\title{
An in vitro Model System for Evaluating Remote Magnetic Nanoparticle Movement and Fibrinolysis
}

This article was published in the following Dove Press journal:

International Journal of Nanomedicine

\author{
Sebastian P Pernal $\mathbb{D}^{1,2}$ \\ Alexander J Willis' \\ Michael E Sabo ${ }^{3}$ \\ Laura M Moore ${ }^{4}$ \\ Steven T Olson (D) $^{5}$ \\ Sean C Morris ${ }^{4}$ \\ Francis $M$ Creighton ${ }^{3}$ \\ Herbert H Engelhard (1D) ${ }^{1,2,6}$ \\ 'The Cancer Center, The University of \\ Illinois at Chicago, Chicago, IL, USA; \\ ${ }^{2}$ Department of Neurosurgery, The \\ University of Illinois at Chicago, Chicago, \\ IL, USA; ${ }^{3}$ UNandUP, LLC, St. Louis, MO,

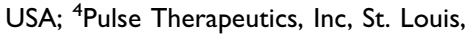 \\ MO, USA; ${ }^{5}$ Department of Periodontics, \\ The University of Illinois at Chicago, \\ Chicago, IL, USA; ${ }^{6}$ Department of \\ Bioengineering, The University of Illinois \\ at Chicago, Chicago, IL, USA
}

Correspondence: Herbert $\mathrm{H}$ Engelhard Department of Neurosurgery, The University of Illinois at Chicago, 912

South Wood St, Chicago, IL 606I2, USA

Tel + I 312 996-4842

Email hengelhard@sbcglobal.net
Background: Thrombotic events continue to be a major cause of morbidity and mortality worldwide. Tissue plasminogen activator (tPA) is used for the treatment of acute ischemic stroke and other thrombotic disorders. Use of tPA is limited by its narrow therapeutic time window, hemorrhagic complications, and insufficient delivery to the location of the thrombus. Magnetic nanoparticles (MNPs) have been proposed for targeting tPA delivery. It would be advantageous to develop an improved in vitro model of clot formation, to screen thrombolytic therapies that could be enhanced by addition of MNPs, and to test magnetic drug targeting at human-sized distances.

Methods: We utilized commercially available blood and endothelial cells to construct $1 / 8$ th inch (and larger) biomimetic vascular channels in acrylic trays. MNP clusters were moved at a distance by a rotating permanent magnet and moved along the channels by surface walking. The effect of different transport media on MNP velocity was studied using video photography. MNPs with and without tPA were analyzed to determine their velocities in the channels, and their fibrinolytic effect in wells and the trays.

Results: MNP clusters could be moved through fluids including blood, at human-sized distances, down straight or branched channels, using the rotating permanent magnet. The greatest MNP velocity was closest to the magnet: $0.76 \pm 0.03 \mathrm{~cm} / \mathrm{sec}$. In serum, the average MNP velocity was $0.10 \pm 0.02 \mathrm{~cm} / \mathrm{sec}$. MNPs were found to enhance tPA delivery, and cause fibrinolysis in both static and dynamic studies. Fibrinolysis was observed to occur in $85 \%$ of the dynamic MNP + tPA experiments.

Conclusion: MNPs hold great promise for use in augmenting delivery of tPA for the treatment of stroke and other thrombotic conditions. This model system facilitates side by side comparisons of MNP-facilitated drug delivery, at a human scale.

Keywords: acute ischemic stroke, biomimetic channel, fibrinolysis, iron oxide nanoparticles, magnetic drug targeting, vascular endothelial cells

\section{Introduction}

Despite significant advances, embolic and thrombotic events (such as ischemic stroke, myocardial infarction, deep venous thrombosis, pulmonary embolism, and peripheral arterial disease) continue to be major causes of morbidity and mortality, worldwide. ${ }^{1-5}$ Considering stroke in the U.S. alone, there are over 795,000 cases annually, with someone having a stroke on average every $40 \mathrm{~s}$. Approximately $87 \%$ of strokes are ischemic and 13\% hemorrhagic, with stroke ranking as the \#5 cause of death, and a major cause of disability, dementia, and depression, costing more than 34 billion dollars annually. Heart disease is the \#1 cause of death in the U.S., with the majority of cases being attributed to ischemic coronary artery disease. ${ }^{6}$ A key therapeutic agent, 
tissue plasminogen activator (tPA) (and its derivatives: alteplase [recombinant], reteplase [modified], tenecteplase [modified]) is a thrombolytic drug which is FDA-approved for the intravenous (IV) treatment of acute ischemic stroke. ${ }^{7,8}$ Thrombolytic therapy with tPA is initiated within 3 to $4.5 \mathrm{hrs}$ after the onset of stroke. ${ }^{9-11}$ TPA is a serine protease which catalyzes the conversion of plasminogen to plasmin, the primary enzyme which degrades fibrin, causing the fibrinolysis which results in clot dissolution. ${ }^{12,13}$ TPA may be used intravenously or locally (i.e., through a catheter) in the treatment of conditions resulting from thrombus formation, such as myocardial infarction, pulmonary embolism, and peripheral arterial disease. ${ }^{14,15}$

Current clinical use of IV tPA is limited by its narrow therapeutic time window, hemorrhagic complications, and suboptimal delivery to the precise location of the thrombus. ${ }^{7,8,13,16,17}$ In computational studies, IV administration of a thrombolytic such as tPA to a thrombus situated within an occluded blood vessel occurs primarily as a function of the length of the occluded vessel and may take 2-3 hrs for peak delivery. ${ }^{18}$ It has been estimated that less than $1 / 100,000 \%$ of drug administered intravenously actually reaches the offending blood clot. ${ }^{18}$ With thrombus lengths exceeding $8 \mathrm{~mm}$, intravenous thrombolytic therapy has a minimal chance of recanalizing the occluded vessel. ${ }^{19}$ While a beneficial agent, augmentation of tPA delivery to the site of the thrombosis is urgently needed, in order to improve its efficacy for re-establishing tissue perfusion. In many acute ischemic stroke patients, such as those with confirmed acute anterior circulation large vessel occlusion, IV tPA combined with catheter-based (i.e., endovascular) therapy has become the first line of treatment. ${ }^{7,20}$ Intravascular catheter placement offers an avenue for directly administering agents designed to augment tPA delivery to the thrombus.

Nanoparticle technology provides compelling strategies for improving the safety and efficacy of thrombus-targeted fibrinolytic therapy. ${ }^{15-17,21-24}$ Magnetic nanoparticles (MNPs) offer a way to improve drug targeting for thrombotic events since they can be directed down "blind alleys" in the vasculature or even tangential to or against prevailing flow, thereby improving drug delivery to the area of obstruction. ${ }^{25,26}$ In the past, while promising at animal-sized distances, ${ }^{27,28}$ successful implementation of magnetic drug targeting in humans has been largely impractical. This has primarily been due to the problem of scale since the attractive force between MNPs and a magnet is inversely proportional to at least the fourth power of the distance. ${ }^{29,30}$ Recently, however, our group reported the development of a model system which allows MNPs to be moved "remotely," over human-sized distances (e.g., 5-30 cm) by means of a rotating permanent magnet. ${ }^{29,31,32}$ This system employs macroscopic lanes which may be coated with endothelial cells, thereby simulating blood vessels, and allows videography for quantification of the velocities of the MNP clusters moving down the lanes. This "remote" induction of MNP movement over such distances has been referred to as "magnetically-enhanced diffusion" or "magneticallyinduced rotation and translation (MIRT)". 29,33,34

In this paper, we report on the next phase of testing of this system, for measuring MNP-mediated delivery of tPA to blood clots in vitro. Our hypothesis was that rotating MNP clusters could be used to enhance delivery of tPA to a clot in a biomimetic channel, even without direct drug conjugation to the MNPs. The model system had three components: 1) standardized MNPs known as microbeads (MBs), 2) a rotating magnet (the "mini-MED"), and 3) custom-designed tissue culture trays containing straight or branched lanes ("MIRT trays"). The lanes of the MIRT trays were filled with fluids such as PBS, culture medium, serum, or citrated whole blood, in order to study the effects of the media on MNP movement. Rabbit endothelial cells were then grown within the lanes of the trays. The effect of MNPs on tPA-mediated fibrinolysis of clotted blood was first studied directly, with a fixed magnet, and then by remote delivery (using the rotating magnet) to thrombi in the biomimetic channels.

\section{Methods}

\section{Magnetic Nanoparticle Microbeads and the Mini-MED System}

Magnetic nanoparticle microbeads (MNPs) and the rotating magnet system ("mini-MED") were provided by Pulse Therapeutics, Inc. (St. Louis, MO). MNPs contain singlecrystalline $\mathrm{Fe}_{3} \mathrm{O}_{4}$ (magnetite) cores $(\sim 40 \mathrm{~nm}$ diameter), are ferrimagnetic, and form agglomerates which act as stir rods in response to a rotating magnetic field. Characterization of these MNPs, including their hysteresis curve and TEM imaging, has recently been reported. ${ }^{29}$ The volume of MNP suspension used in most experiments described here was $20 \mu \mathrm{L}$. This was from a stock solution of $25 \mathrm{mg} / \mathrm{mL}$, so typically $0.5 \mathrm{mg}$ of MNPs was used for each determination (i.e., per lane as described below). The "mini-MED" contains a neodymium-boron-iron permanent magnet (see US patents $8,313,422,8,308,628$, and $8,529,428$ ), which spins rapidly (3 $\mathrm{Hz}$ ), causing agglomerated MNPs to counter-rotate (like meshing gears), and move by means of surface traction. Rotating MNP clusters have also been referred to as "aggregates", 
although more technically they are "agglomerates". These MNPs have been designed to be optimized with respect to their lack of toxicity, and kinetic response to the mini-MED. Bio-compatibility testing of the MNPs was conducted under ISO 10993, as a blood-contacting medical device, with a duration of greater than 30 days. Under ambient conditions even without exposure to a magnet, the MNPs form linear structures, as seen in Figure 1A.

\section{Production of "Magnetically-Induced Rotation and Translation" (MIRT) Trays}

Trays for testing the movement of MNPs by the mini-MED were designed in Inventor Professional 2017 (Autodesk, Mill Valley, CA), with dimensions to be compatible with standard 96-well plate readers (namely, $127.8 \mathrm{~mm}$ length, $85.5 \mathrm{~mm}$ width, $13.0 \mathrm{~mm}$ height). While the lanes of the trays can be made according to a wide variety of designs, two basic designs were used in the experiments reported here. For the "straight lane" tray, each lane was $1 / 8$ th inch $(3.175 \mathrm{~mm})$ wide, similar in size to a portion of a human cerebral or coronary artery. The straight lane design, in which the tray has 8 lanes, allows direct comparisons to be made between different types of magnetic particles, or different transport media, provided that the magnetic field effect is homogeneous. Tray lanes were separated by $9 \mathrm{~mm}$ center to center, with the beginning of the first lane being designed to match the 96-well “A1" position. Utilizing Inventor Professional's computer-aided manufacturing (CAM), drill specifications were set for the milling of an optically clear, cast acrylic rectangular bar (McMaster-Carr Supply Co., Elmhurst IL). To produce the trays, g-code was uploaded to an Othermill Pro milling machine (Other Machine Co., Berkeley, CA). The acrylic bar was milled first with a $1 / 8$ th inch flat bit for initial cutting and removal of bulk material. Then, a $1 / 8$ th inch ball-end bit was used with lubricant to finish the trays, in order to produce smooth lanes with rounded bottoms.

For the "branched lane" tray, the same material and overall dimensions were used. The branching pattern includes six "arteries" with 5 bifurcations in two dimensions. The "beginning artery" (representing where a catheter could potentially deposit MNPs and drug, such as in the internal carotid artery) is centered at position D1 of a standard 96-well plate, and has a width of $1 / 2$ inch $(12.7 \mathrm{~mm})$. At the first bifurcation, the lane width decreases to $3 / 8$ th inch $(9.525 \mathrm{~mm})$, while the second bifurcation further reduces the lane width to $1 / 4$ inch $(6.35 \mathrm{~mm})$. For the sake of simplicity for the prototype, branches at 45 degrees (with rounded edges) were used. The final trays were washed and cleaned with distilled water, removing any milled acrylic residue. When used for tissue culture, trays were sterilized overnight using ethylene oxide, or with $100 \%$ ethanol and UV light. Successive sterilizations, of ethylene oxide or ethanol and UV light, did not alter the structure or integrity of the MIRT tray. Representative trays showing the straight lane and branched lane designs - are seen in Figure 1B and C. Figure 1D shows a computerized tomography (CT) image of a portion of the straight lane tray.

\section{Video Analysis of MNP Velocity; Tray Positions}

Digital videos of MNP aggregates (or agglomerates) being moved by the mini-MED in the MIRT trays were recorded using an Olympus SZ-12 camera (Center Valley, PA). Videos were recorded either in an "above" or "below" imaging position, with the MIRT tray on a grid indicating each centimeter of distance from the starting position (origin) of the MNPs. "Above" imaging was performed for transparent liquids, such as phosphate-buffered saline (PBS) or cell culture media. "Below" imaging - with the tray below the miniMED and the camera below the tray - was required for opaque liquids, such as whole blood or skim milk. This is because the MNP aggregates are more easily seen at the bottom of the lanes. A diagram illustrating the different tray positions used for this paper is given in Figure 2A. The origin (or starting point) for the MNPs (from the vantage point of this figure) was always to the left. The MNPs moved to the right, whether above or below the magnet, in response to the rotation of the mini-MED. Digital recording analysis was performed using MATLAB's Video Viewer application (MathWorks, Natick, MA). As the leading edge of the MNP mass passed each marked centimeter, the frame number was recorded. The difference in frame number was determined between centimeters. The frame rate of the camera (frames/sec) was divided by the difference in frames per marked centimeter (frames $/ \mathrm{cm}$ ) producing a velocity reading (in $\mathrm{cm} / \mathrm{sec}$ ) of the MNP movement over each centimeter. A photograph of the mini-MED with the MIRT tray in the "offset above" position is shown in Figure 2B. This perspective is rotated 90 degrees clockwise from that of Figure 2A, and shown from above.

\section{MNP Translation (with the Mini-MED) and Velocity Determinations}

MNP velocities were initially measured in a cell-free system using $1 \mathrm{~mL}$ (per lane) of 5 different solutions: PBS, Dulbecco's Modified Eagle's Medium (DMEM), fetal 
A

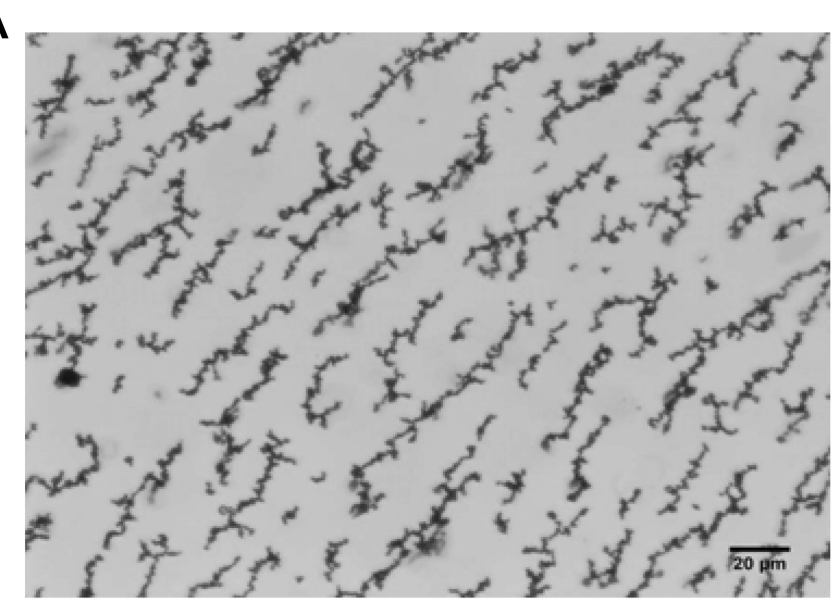

B

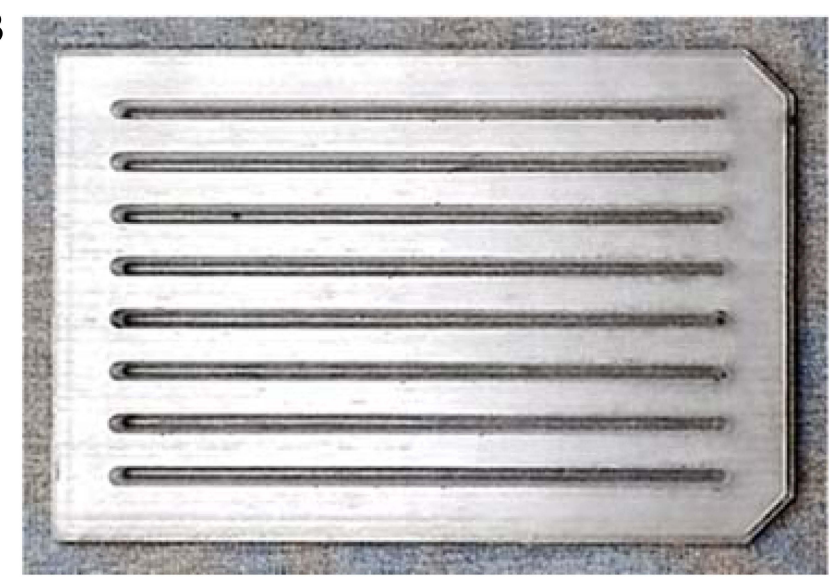

C

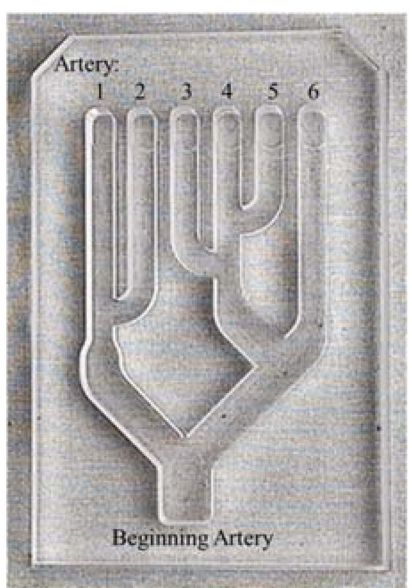

D

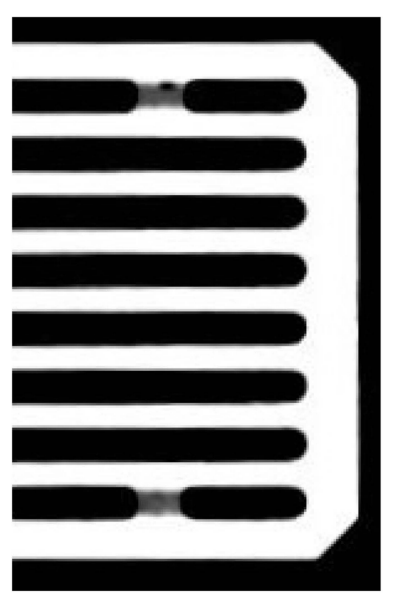

Figure I (A) Photomicrograph of linear structures of MNPs formed without exposure to a magnet. (B) Photograph of the original MIRT tray, with straight lanes allowing for direct comparison of different experimental conditions such as MNP concentration, fluid composition, and cellular lining of the lanes. (C) Photograph of a representative MIRT tray with branched lanes (rotated and at reduced magnification), which allows for the study of MNP distribution along various tributaries. (D) Computerized tomography image of a portion of the straight lane MIRT tray. 


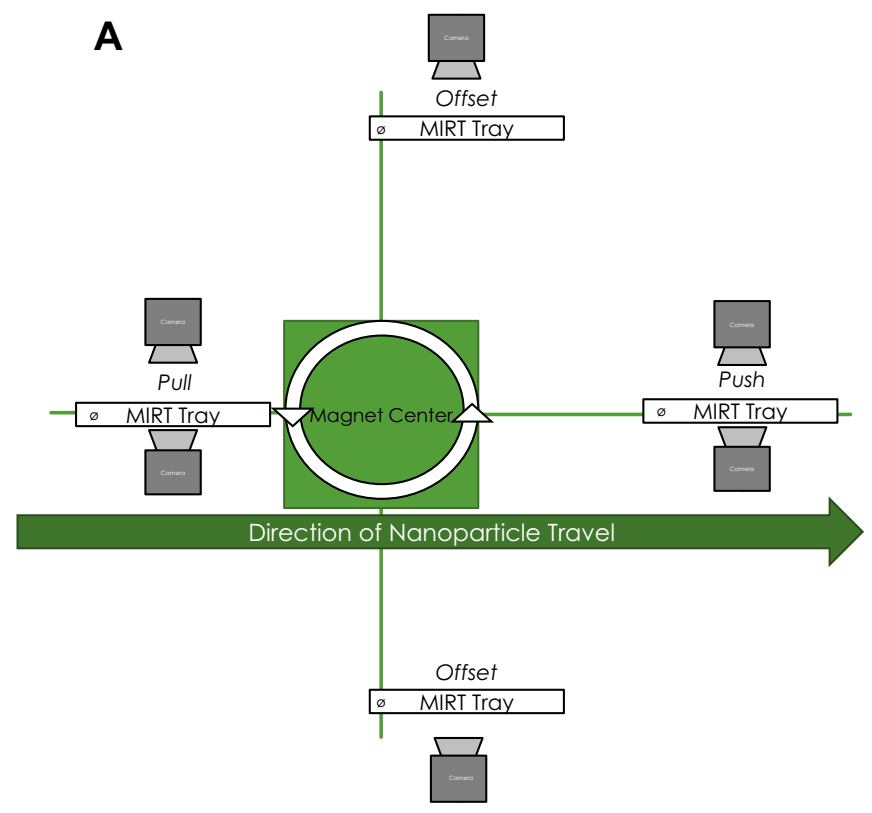

B

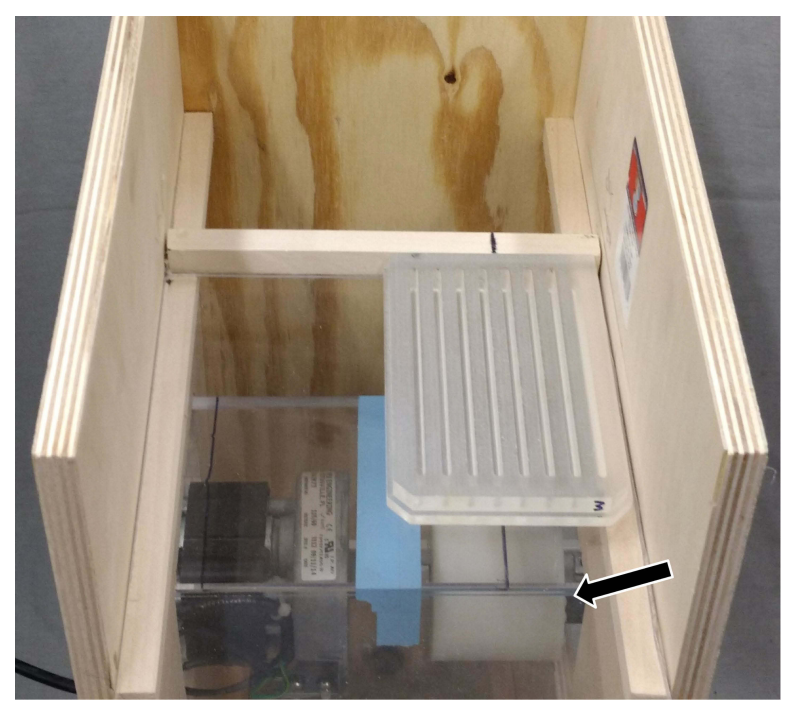

Figure 2 (A) Illustration showing the various positions of the MIRT tray with respect to the rotating magnet (mini-MED), and the direction of MNP travel. "ø" indicates the origin or start position for MNPs. (B) Photograph of the MIRT tray in the offset above position, $20 \mathrm{~cm}$ over the mini-MED. The location of the rotating permanent magnet is shown by the arrow. This perspective is rotated $90^{\circ}$ and shown from above. The MNPs move remotely (i.e., at a distance from the magnet) due to the rotation of the clusters, and surface traction.

bovine serum (FBS), bovine skim milk, and rabbit whole blood. Twenty $\mu \mathrm{L}$ of $25 \mathrm{mg} / \mathrm{mL}$ MNP solution was added to the starting points (i.e., origins) of the lanes of the tray. MNPs were pre-magnetized by placing the tray (with MNPs at their starting points) at the "pull" side of the magnet, which consolidated the MNPs at each lane origin. The tray was then mounted at the position of interest (as seen in Figure 2A) so that the middle of the imaged lanes would be along the central plane of the magnet. MNP translation in the branched lane MIRT tray was studied using $8 \mathrm{~mL}$ of PBS, which filled all the branches. Fifty $\mu \mathrm{L}$ of $25 \mathrm{mg} / \mathrm{mL}$ MNP solution was added to the "beginning artery" and magnetized as described. The branched lane tray was studied in the $20 \mathrm{~cm}$ pull position and the center of the beginning artery was used as the fulcrum for rotation. In the 0 degree position, the tray was not pivoted and was aligned along the center of the plane of the rotating magnet. In the +45 degrees position, the tray was pivoted counterclockwise 45 degrees while maintaining the center of the beginning artery along the center of the magnet. In contrast, in the -45 degrees position, the tray was pivoted clockwise 45 degrees on the same fulcrum.

Quantification of MNPs at the end positions of the branched tray was determined using spectrophotometry. To do this, MNPs at the ends of the lanes (after running the mini-MED) were pipetted into labeled $1.5 \mathrm{~mL}$ centrifuge tubes, then centrifuged for $10 \mathrm{~min}$ at $6000 \mathrm{rpm}$. The supernatant was carefully removed, and the MNP pellet was resuspended in $500 \mu \mathrm{L}$ PBS, sonicated, and then vortexed for $30 \mathrm{sec}$. One hundred $\mu \mathrm{L}$ was pipetted into 3 wells of a 96-well plate for each individual artery versus a PBS control. Absorbance was measured at 340 and $777 \mathrm{~nm}$ in order to determine MNP concentration. MNP localization into each branch was therefore determined, and shown as a percentage of the original quantity placed at the origin.

\section{Rabbit Endothelial Cells and Fluorescence Microscopy}

Rabbit aortic endothelial cells (Cell Biologics, Chicago, IL) were maintained using standard tissue culture technique $\left(37^{\circ} \mathrm{C}\right.$, $5 \% \mathrm{CO}_{2}$ ) in basal medium supplemented with $0.1 \%$ VEGF and EGF, 1\% L-glutamine, antibiotic-antimycotic solution, and 2\% FBS (Cell Biologics, Chicago, IL). For MIRT tray studies of MNP velocities over cells, $10^{5}$ cells were dispersed into lanes of the sterilized MIRT tray, which were pre-coated with $1 \mathrm{~mL}$ of $0.1 \%$ gelatin. Cells were grown for $48 \mathrm{hrs}$ (until confluent) before further experimentation. Immediately prior to determinations of MNP velocities, the basal medium was replaced with PBS. For fluorescent staining, cells were washed 
with phosphate-buffered saline (PBS) before being fixed with $4 \%$ paraformaldehyde for $10 \mathrm{~min}$ at room temperature, then washed again. A solution of phalloidin in PBS (1:40 v/v) was applied to the cells for $20 \mathrm{~min}$ at $37^{\circ} \mathrm{C}$ (in the incubator), followed by addition of an aliquot of Hoescht 33342 (1:1000 v/v; Sigma-Aldrich, St. Louis, MO) for $10 \mathrm{~min}$ again at $37^{\circ} \mathrm{C}$. Cells were imaged using an EVOS FL Auto 2 microscope (Fisher Scientific, Waltham, MA).

\section{Thrombus Formation, Absorbance Assay, and tPA/MNP Treatments - Static Studies}

Whole rabbit blood, anticoagulated by citrate, was obtained from Hemostat Laboratories (Dixon, CA). Using a $1.5 \mathrm{~mL}$ centrifuge tube, $1 \mathrm{~mL}$ of whole anticoagulated rabbit blood was combined with $50 \mu \mathrm{L}$ of $1 \mathrm{M}$ $\mathrm{CaCl}_{2}$ (Fisher Scientific, Hampton, NH). The centrifuge tube was moved back and forth lightly to mix the contents, then incubated at $37^{\circ} \mathrm{C}$ for $20 \mathrm{~min}$, allowing the blood to coagulate. $^{35}$ For the clot absorbance assay, clots were gently retrieved from centrifuge tubes using tweezers, then washed in a room temperature PBS bath to remove unclotted blood from the surface. Formed clots (red thrombi) were trimmed to the size of a well, then placed at the bottom of the wells in a 96-well plate (Corning, Tewksbury, MA). Rabbit tPA (Abcam, Cambridge, UK) and/or MNPs were added to clots in order to determine their effect on absorbance readings, versus untreated controls. Ten $\mu \mathrm{g}$ of tPA, and/or 0.025 or $0.25 \mathrm{mg}$ of MNPs (1 or $10 \mu \mathrm{L}$ of $25 \mathrm{mg} / \mathrm{mL}$ MNP stock solution) were added to each well. After $2 \mathrm{hrs}$ of incubation at $37^{\circ} \mathrm{C}$, plates were analyzed using a SPECTRAmax 340PC (Molecular Devices, Inc., San Jose, CA), utilizing wavelengths from 600 to $700 \mathrm{~nm}$. Tweezers were used in each well to remove any clots that were remaining after the treatment period.

In complementary experiments, fibrin clots were formed in $3.4 \mathrm{~mm}$ diameter glass tubes as per Viennet et al. ${ }^{36}$ Each fibrin clot was formed using $50 \mu \mathrm{L}$ of a clotting mixture at $\mathrm{pH} 7.4$ containing: gelatin $0.05 \% \mathrm{w} / \mathrm{v}$; human plasminogen, $3.5 \mathrm{U} / \mathrm{ml}$; human thrombin, $30 \mathrm{U} / \mathrm{ml}$; and bovine fibrinogen, $20.7 \mathrm{mg}$ clottable protein $/ \mathrm{ml}$, in PBS. $\varepsilon$-aminocaproic acid (EACA), an inhibitor of fibrinolysis, was added to the clotting mixture in order to achieve a $3 \mathrm{mM}$ concentration for the fibrin clots, and $4 \mathrm{mM}$ concentration for the whole blood clots. Following clot formation at $37^{\circ} \mathrm{C}$ for $10 \mathrm{~min}$, tubes were cooled to room temperature, sealed with Parafilm and stored overnight at $4^{\circ} \mathrm{C}$ prior to use. On the day of the assay, $130 \mu \mathrm{L}$ of 0.1
M phosphate buffer ( $\mathrm{pH} 7.4$ ) containing $20 \mathrm{U} / \mathrm{ml}$ plasminogen, $1200 \mathrm{U} / \mathrm{ml}$ alteplase and test reagents (as indicated) were placed on top of the clot. For clots with MNPs, $20 \mu \mathrm{g}$ were used. Tubes were placed in a custom-built lightbox with backillumination of the tubes in order to capture their images. Clot lysis was allowed to proceed at room temperature and quantified by tracking the regression of the clot/buffer interface using the camera. The rate of lysis was linear for up to $4 \mathrm{hrs}$. Whole blood clots were similarly formed in $3.4 \mathrm{~mm}$ glass tubes using a method based on that of Mann et al. ${ }^{37} \mathrm{CaCl}_{2}$ was added (final concentration $12 \mathrm{mM}$ ) to $50 \mu \mathrm{L}$ pooled citrated human blood (Innovation Research, MI), and clotting allowed to proceed for $30 \mathrm{~min}$ at $37^{\circ} \mathrm{C}$. After cooling to room temperature, the tubes were sealed using Parafilm and stored at $4^{\circ} \mathrm{C}$ prior to lysis assay within 3-7 days. On the day of the assay, $130 \mu \mathrm{L}$ of pooled human plasma containing added $20 \mathrm{U} / \mathrm{ml}$ human plasminogen and the test reagents was placed above the clots, and the tubes were placed in the lightbox, with lysis measured as described above.

\section{TPA/MNP Treatments in the MIRT Tray - Dynamic Studies}

These experiments were designed to determine whether or not MNPs could be used to deliver a drug (here, tPA) to a clot by means of enhanced fluid transport, in the absence of drug conjugation to the nanoparticle itself. Thrombi from whole rabbit blood were made as described above (static studies), gently retrieved using tweezers, trimmed to size, washed, and positioned in the lanes of the MIRT tray. Studies were performed with the straight lane MIRT tray in the $20 \mathrm{~cm}$ offset position, with and without endothelial cells. For studies of clots in the MIRT tray, each lane to be tested was prepared with two $5 \mathrm{~mm}$ agarose blocks, one placed $6-8 \mathrm{~cm}$ from the origin, and another after an additional $0.5 \mathrm{~cm}$ distance, so that clots would be approximately $0.5 \mathrm{~cm}$ wide. Between the agarose blocks, $300 \mu \mathrm{L}$ of whole rabbit blood (without calcium) was added before clot placement, to ensure sealing of the clot in the lane. Blood escaping outside of the agarose blocks was aspirated to avoid interference with the MNPs. An additional $50 \mu \mathrm{L}$ of $1 \mathrm{M}$ $\mathrm{CaCl}_{2}$ was added to ensure clotting.

The MIRT tray with clots was placed in an incubator at $37^{\circ} \mathrm{C}$ for $1 \mathrm{hr}$. After incubation, agarose blocks were removed and ends of the clots were washed with $200 \mu \mathrm{L}$ of PBS. If PBS passed through a clot, the clot was discarded. MNPs, tPA, PBS, and gel packs were pre-warmed in a water bath set to $40^{\circ} \mathrm{C}$. Ten micrograms of tPA was mixed with $20 \mu \mathrm{L}$ of MNPs $(25 \mathrm{mg} / \mathrm{mL})$ and vortexed 
together for $30 \mathrm{sec}$. Pre-warmed gel packs were placed underneath the MIRT tray, in order to maintain a physiologic temperature. MNPs alone or mixed with tPA were added to the MIRT tray origins and magnetized, as usual. The mini-MED was then activated for 70-100 min per experiment to accurately determine the effect of MNPs (with or without tPA) on the clots. Fibrinolysis was visually determined to have occurred, if the lane preceding the thrombus became positive for blood, resulting from the release of red blood cells (which had previously been encased in fibrin).

For confirmation of clot lysis, media was aspirated carefully to minimize interference with the clot. One milliliter of PBS was added to each lane, then $20 \mu \mathrm{L}$ of MNPs $(25 \mathrm{mg} / \mathrm{mL}$ ) was added to the tray lanes at their origins, and allowed to run as usual. This "proof of clot lysis" test only required running the mini-MED for $5 \mathrm{~min}$. "Complete clot lysis" was confirmed if MNPs were able to pass freely (i.e., within the expected time frame of approximately 1 min) through the previous location of the clot. "Partial clot lysis" was present if the MNPs took longer than the expected amount of time (for instance: $5 \mathrm{~min}$.) "Incomplete/no clot lysis" was present if the MNPs were unable to run through the previous site of the clot. Clot lysis efficiency was defined as the number of these events over the total number of clot lysis attempts.

\section{Data Display and Tests of Statistical Significance}

Data (e.g., the velocity curves) are expressed as mean and standard deviation (mean \pm SEM) with $n$ values as given in the figure legends. Statistical significance for the velocity and absorbance experiments was determined using two-factor ANOVA with replication. For rates of clot lysis in the static studies, statistical significance was tested using ANOVA (Sidak's multiple comparisons test). Statistical significance for fibrinolysis and the clot lysis events in the dynamic studies was determined using a Z-score for proportions. A value of $p<0.05$ was considered statistically significant.

\section{Results}

\section{Movement of MNPs in the Straight Lane MIRT Tray Without Cells: Effect of Different Media and Tray Positions on MNP Velocity}

The mini-MED, in a stationary position, causes the MNPs to move along surfaces, including the lanes of the MIRT tray, and this can be used to facilitate comparisons between different experimental conditions or drug formulations. Velocities of MNPs through media of varying viscosity and protein content were compared. Media included PBS, DMEM, FBS, skim milk, and whole blood. A photograph of MNPs at the origins (starting points) of lanes filled with citrated whole blood, FBS, and DMEM is seen in Figure 3A. A photograph of MNPs running through $\mathrm{PBS}$ in response to the miniMED (one lane) is seen in Figure 3B. The arrow indicates the leading edge of the MNPs. The leading edges of MNPs were used for velocity determinations, which were made in centimeter increments as MNPs progressed down the lanes. Due to the opacity of blood, the "offset below" position (with the tray $20 \mathrm{~cm}$ below the magnet center, and the camera filming from below the tray) was used for these experiments. This position is illustrated at the bottom of Figure 2A.

As anticipated, the velocities of the MNPs were different, in different types of fluids. The average velocity across the entire tray (at the $20 \mathrm{~cm}$ offset below position) in PBS was found to be $0.18 \pm 0.01 \mathrm{~cm} / \mathrm{sec}$. In DMEM, the MNPs had an average velocity $0.180 \pm 0.02 \mathrm{~cm} / \mathrm{sec}$. In FBS and skim milk, the average velocity was slower; namely $0.10 \pm 0.02 \mathrm{~cm} / \mathrm{sec}$, and $0.06 \pm 0.01 \mathrm{~cm} / \mathrm{sec}$, respectively. In citrate-anticoagulated rabbit whole blood, the average velocity was even slower, namely $0.030 \pm$ $0.004 \mathrm{~cm} / \mathrm{sec}$. Serum from different species demonstrated MNP velocities similar to FBS. Whole blood has a greater viscosity, and therefore imparts a greater drag on the spinning MNP clusters. MNP clusters may dissociate in more viscous fluids. Even though the velocity was less, the leading edge of the MNPs traversed the entire $10.5 \mathrm{~cm}$ length of the tray in $360.4 \pm 11.5 \mathrm{sec}$, when moving through whole blood. Velocities over each $\mathrm{cm}$ interval along the lanes are displayed in Figure 3C. The relationship between fluid viscosity and average MNP velocity (in the offset below position) is seen in Figure 3D.

Changing the position of the MIRT tray relative to the mini-MED also changed the velocities of the MNPs. Velocities of MNPs (in PBS and blood) at two different key positions with respect to the mini-MED are shown in Figure 4A and B. Figure 4A shows PBS and whole blood data from the offset below position, with a scale change for the $y$-axis. Figure 4B shows the greater MB velocities in the pull position, in which the MNPs are running directly toward the magnet, being affected by rotational traction and accelerated by magnetic attraction. This resulted in the greatest velocity closest to the magnet, 
A

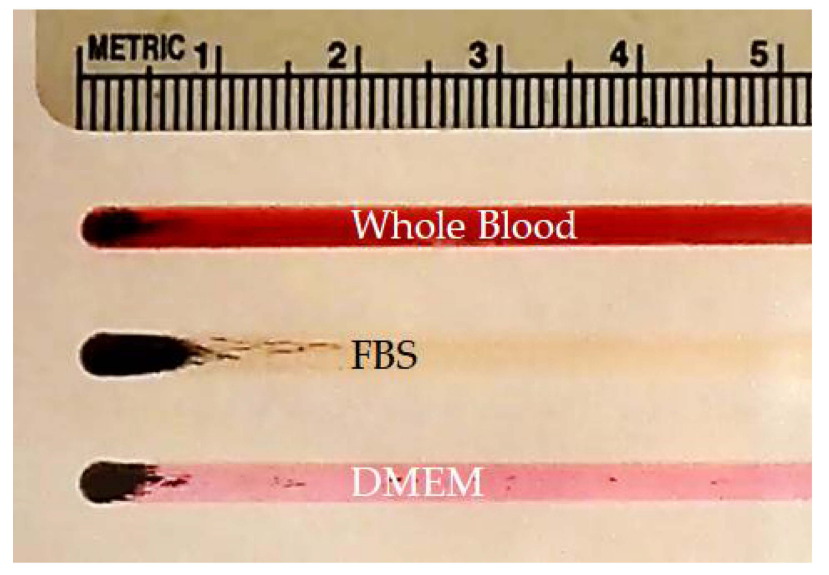

B
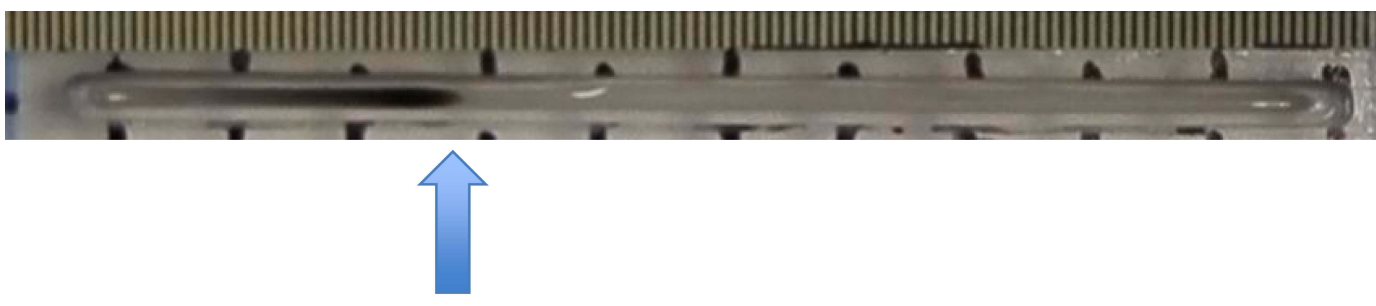

C
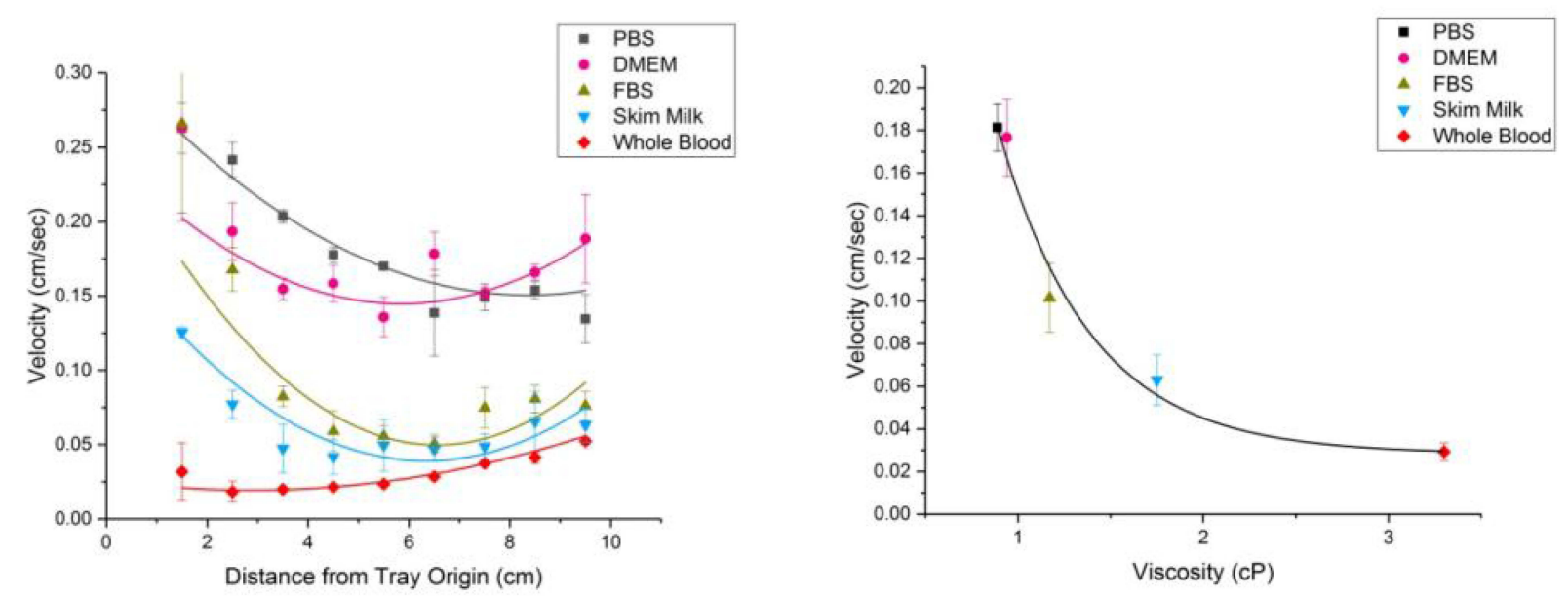

Figure 3 (A) Photograph of MNPs at the starting position (origins) of the straight-laned MIRT tray, in three different fluids, from top to bottom: whole blood, FBS, and DMEM. (B) Photograph of MNPs (with advancing edge at the arrow, in PBS) moving down the tray in response to the action of the mini-MED. (C) Velocity of MNPs in the MIRT tray (y-axis) vs. distance from tray origin (x-axis) for five media (PBS, DMEM, 100\% FBS, skim milk, and whole blood) in the $20 \mathrm{~cm}$ offset below position ( $\mathrm{n} \geq 3$ ). (D) Correlation between the viscosity of media (PBS, DMEM, 100\% FBS, skim milk, and whole blood) and the average velocity in the $20 \mathrm{~cm}$ offset below position.

namely $0.76 \pm 0.03 \mathrm{~cm} / \mathrm{sec}$. MNPs in the push position stop moving at a distance of about $27 \mathrm{~cm}$ away from the magnet center in whole blood, and at $29 \mathrm{~cm}$ in PBS. MNP velocity was slightly greater when moving through PBS in the offset above position in comparison to the offset below position (at the same distance from the magnet), likely due to the increased surface friction (traction) produced when the tray and particles were above the magnet. 


\section{Effect of Tray Lane Branching and Magnet Alignment on MNP Distribution}

MNP movement was studied in the branched lane tray in the pull position to model the effect of conduit branching on MNP distribution (such as within an arterial tree), also using video photography. The set-up is depicted in Figure 5A. The average MNP velocity throughout the branched lane tray (in the neutral position) was found to be $0.21 \pm 0.02 \mathrm{~cm} / \mathrm{sec}$, ranging from $0.10 \pm 0.01$ to $0.35 \pm 0.01 \mathrm{~cm} / \mathrm{sec}$ depending on the path of the MNPs through the branches of the tray. Changing the rotation of the branched lane MIRT tray relative to the mini-MED (also in the pull position) provided results that would be anticipated, given an understanding of the surface walking phenomenon of MNPs. The tray in the original position ( 0 degrees) gave a final MNP distribution that favored MNPs moving to the end of "artery" 4, and approximately equivalent MNP distributions in "arteries" 1 and 2 (as seen in the central pane). In the +45 degree position, all MNPs were directed to the end of "artery" 6 with an average velocity of $0.38 \pm 0.01 \mathrm{~cm} / \mathrm{sec}$; and in the -45 degree position, all of the MNPs were directed to the end of "artery" 1 with an average velocity of $0.30 \pm 0.01 \mathrm{~cm} / \mathrm{sec}$. The different amounts of MNPs in each lane and position (as quantified spectrophotometrically) are seen in Figure 5B. The difference in the average MNP end concentrations of artery 1 in the 0 versus -45 degree position was found to be statistically significant $(p<0.005)$. The difference in the average MNP end concentrations of artery 6 in the 0 versus +45 degrees position was also statistically significant $(\mathrm{p}<0.005)$. These data emphasize the extent to which orientation of the rotating magnetic field plays a role in the movement of MNPs in conduits in which fluid flow is stagnant, such as in an obstructed artery, or in the MIRT tray.

\section{Effect of an Endothelial Monolayer on MNP Velocity}

Rabbit vascular endothelial cells were seeded and grown in the lanes of the sterilized MIRT tray (for $48 \mathrm{hrs}$ until confluent) in order to construct a primitive model of a large blood vessel (3.175 $\mathrm{mm}$ diameter). Figure 6A shows rabbit vascular endothelial cells that were grown in the straight lane MIRT tray for $24 \mathrm{hrs,} \mathrm{then} \mathrm{fixed} \mathrm{and}$ stained for F-actin (green) and DNA (blue). Cells appear more blurry towards the right and bottom edge of the photo due to the curvature of the lane, which takes the cells outside of the focal plane of the microscope. Viability of endothelial cells grown in the straight lane MIRT tray was found to be excellent up to $120 \mathrm{hrs}$ (the longest time studied), whether 25,$000 ; 50,000$ or 100,000 cells were used for the initial seeding. Cells could also be grown and photographed in the branched lane tray. Velocity of MNPs in the straight lane MIRT tray through PBS and over rabbit endothelial cells versus in PBS alone (without cells) was then studied. Data are shown in Figure 6B for the $20 \mathrm{~cm}$ offset above position. Addition of vascular endothelial cells to the lanes of the MIRT tray was found to slow the velocity of the MNPs, from an average of $0.27 \pm$ $0.02 \mathrm{~cm} / \mathrm{sec}$ without the monolayer to $0.21 \pm 0.01 \mathrm{~cm} / \mathrm{sec}$ over the monolayer of cells, in PBS at the offset above position $(p<0.005)$.

\section{Blood Clot Production, Absorbance Assay Results, and MNP/tPA Treatment - Static Studies}

In order to reproducibly form small clots, different concentrations of calcium chloride (ranging from 0.1 to $1 \mathrm{M}$ ), and varying volumes of calcium (ranging from 40 to $500 \mu \mathrm{L}$ ) and citrate-anticoagulated rabbit whole blood, were tested. For making a "standard clot", the combination of $50 \mu \mathrm{L}$ of $1 \mathrm{M}$ calcium chloride added to $1 \mathrm{~mL}$ of blood was found to be best for subsequent experiments. Blood clots of $0.8 \mathrm{~cm}^{3}$ were able to be reproducibly produced for 96-well plates and the MIRT trays. Clots (with and without MNPs) could be differentiated from unclotted whole blood by spectrophotometry, both in the plates and in the trays. As seen in Figure 7A, the absorbance of clotted whole blood was greater than the absorbance of unclotted whole blood over multiple wavelengths, but particularly at $640-720 \mathrm{~nm}$. This is as expected since a denser structure should not allow as much light to be transmitted. The addition of MNPs to clots (demonstrated at $2.5 \mathrm{mg} / \mathrm{ml}$ and $25 \mathrm{mg} / \mathrm{ml}$ in Figure 7A) was seen to produce additional absorbance, and this could be used to differentiate clots with or without MNPs.

Clot softening and lysis by $10 \mu \mathrm{g}$ of tPA per well in 96well plates were then demonstrated. This effect is seen in Figure 7B, illustrating that the shape and structure (i.e., morphology) of the extracted clots was different, depending on tPA treatment. Clots without treatment were rigid, while the tPA-treated clots were fluid, and malleable. Solid clots could be removed from the plates with a tweezers. This fact was reflected in the absorbance reading at 640 $\mathrm{nm}$, as seen in Figure 7C. TPA-treated clots produced absorbance readings similar to that of the whole blood control $(p=0.96)$. Clots made from freshly obtained rabbit blood yielded results similar to those from commercially 


\section{A OFFSET BELOW POSITION}

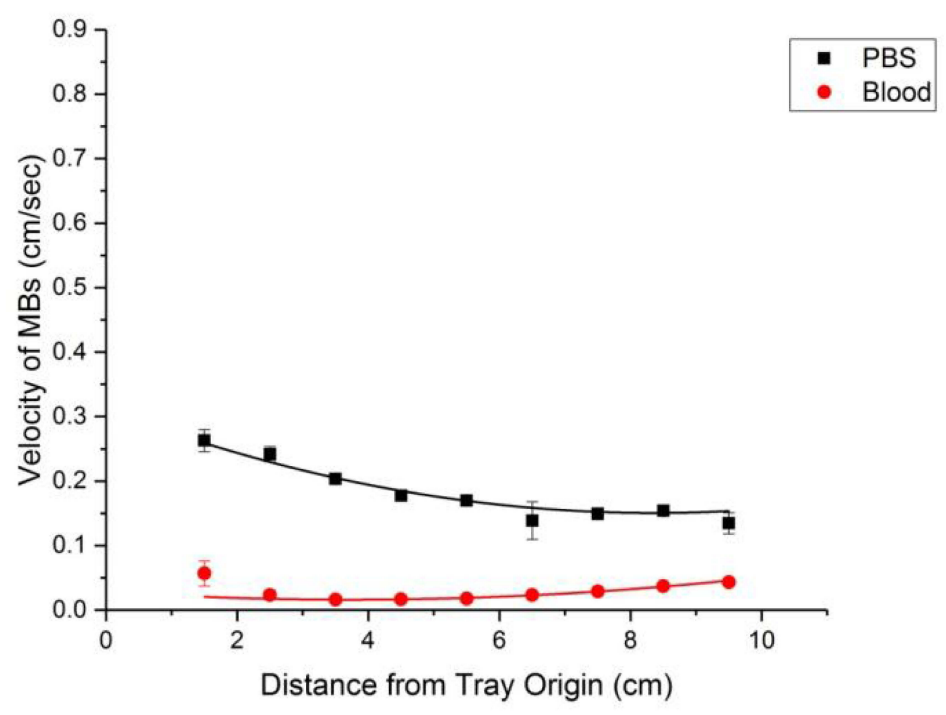

\section{B PULL POSITION}

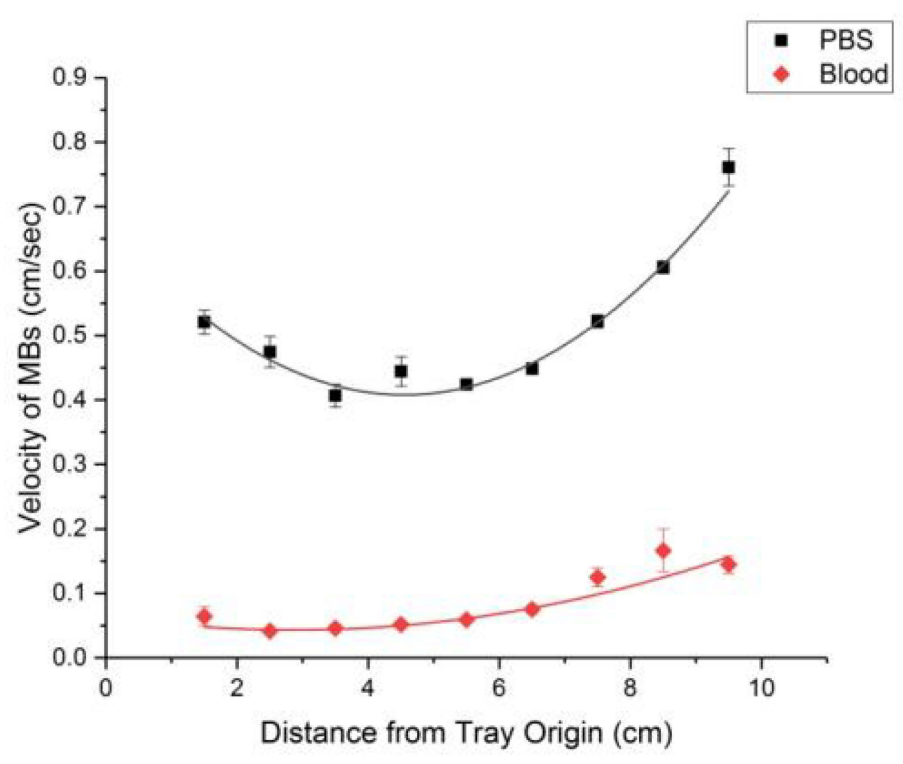

Figure 4 A comparison of MNP velocities in the MIRT tray, traveling through PBS versus unclotted whole blood, using two different tray positions ( $\mathrm{n}=9$ ). $(\mathbf{A}) 20 \mathrm{~cm}$ offset below position (B). $20 \mathrm{~cm}$ pull position. Note that MNPs in the "pull" position in PBS and closest to the magnet have the greatest velocity.

available blood. Commercially available blood, however, was found to be more convenient and yield more consistent results.

The rates of fibrin clot lysis, and whole blood clot lysis, were measured under different experimental conditions, as shown in Figure 7D and E, respectively. These experiments were performed in tubes. Under control conditions (blank, MNPs + magnet, and tPA + EACA + MNPs + magnet), the rate of clot lysis (of fibrin or blood) was negligible. When tPA was added alone, with or without MNPs, some lysis did occur, as seen. However, when tPA was added with MNPs and the magnet together - either on fibrin clots or whole blood clots - the rate of lysis was much faster, approximately $6 \mathrm{~mm} / \mathrm{hr}$, and this finding was 
A

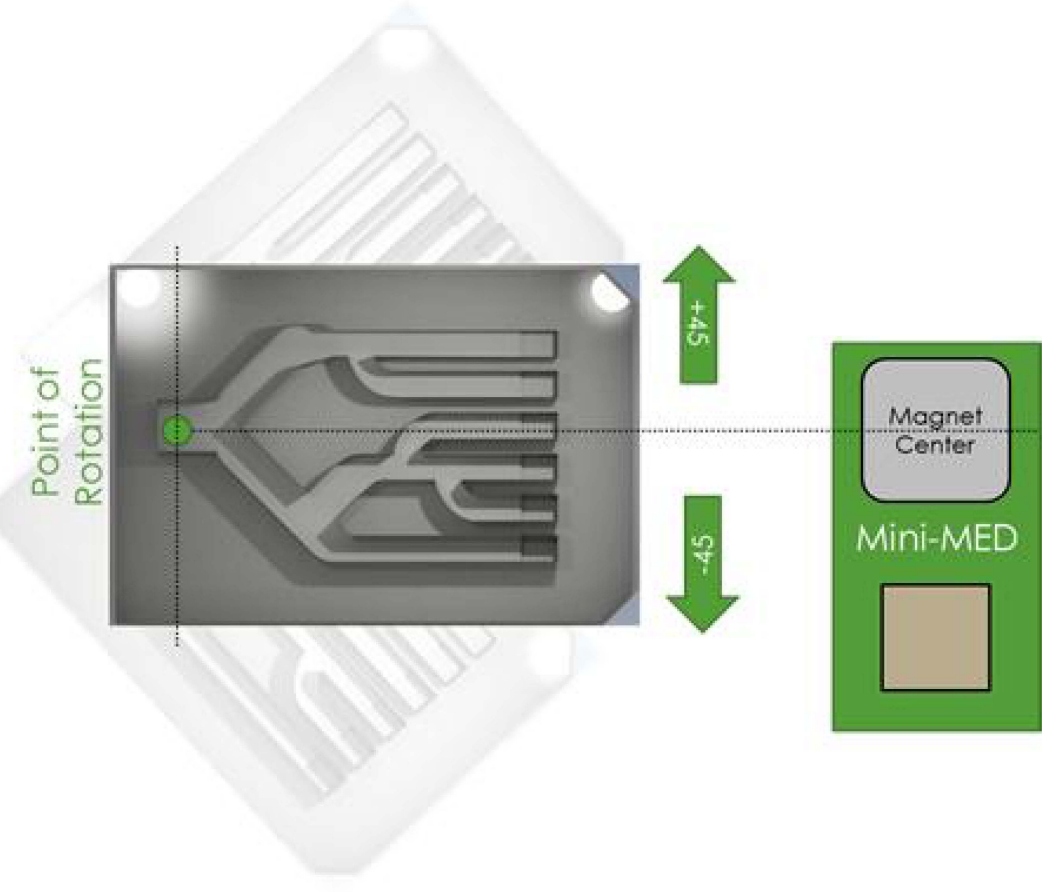

B
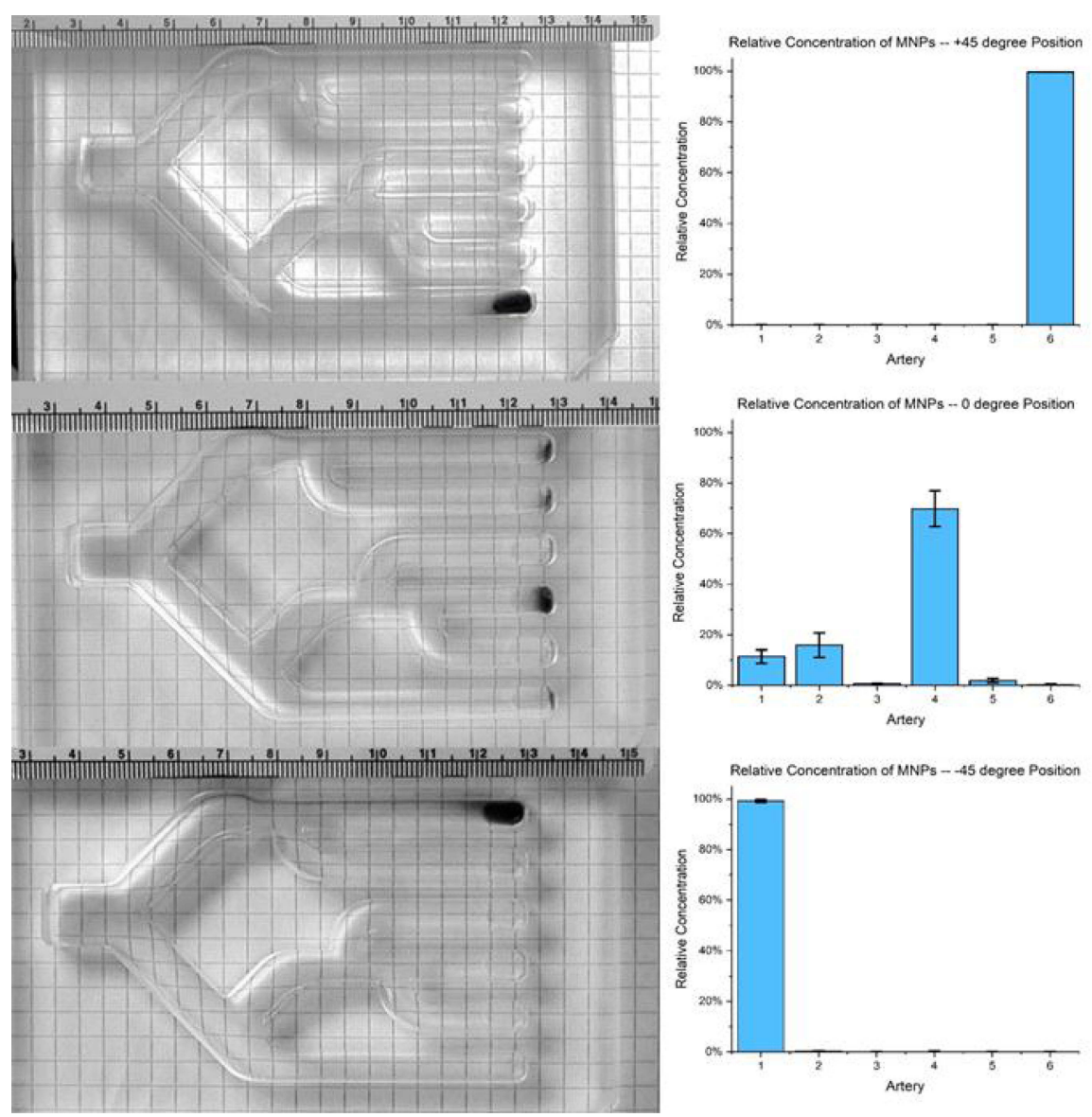

Figure 5 (A) Illustration of branched lane MIRT tray in pull position with rotation at $+45,0$, and -45 degrees with respect to the center of the rotating magnet. (B) Relative final concentrations of MNPs after changing the orientation of the branched lane MIRT tray in the pull position $(n \geq 3)$. 
A

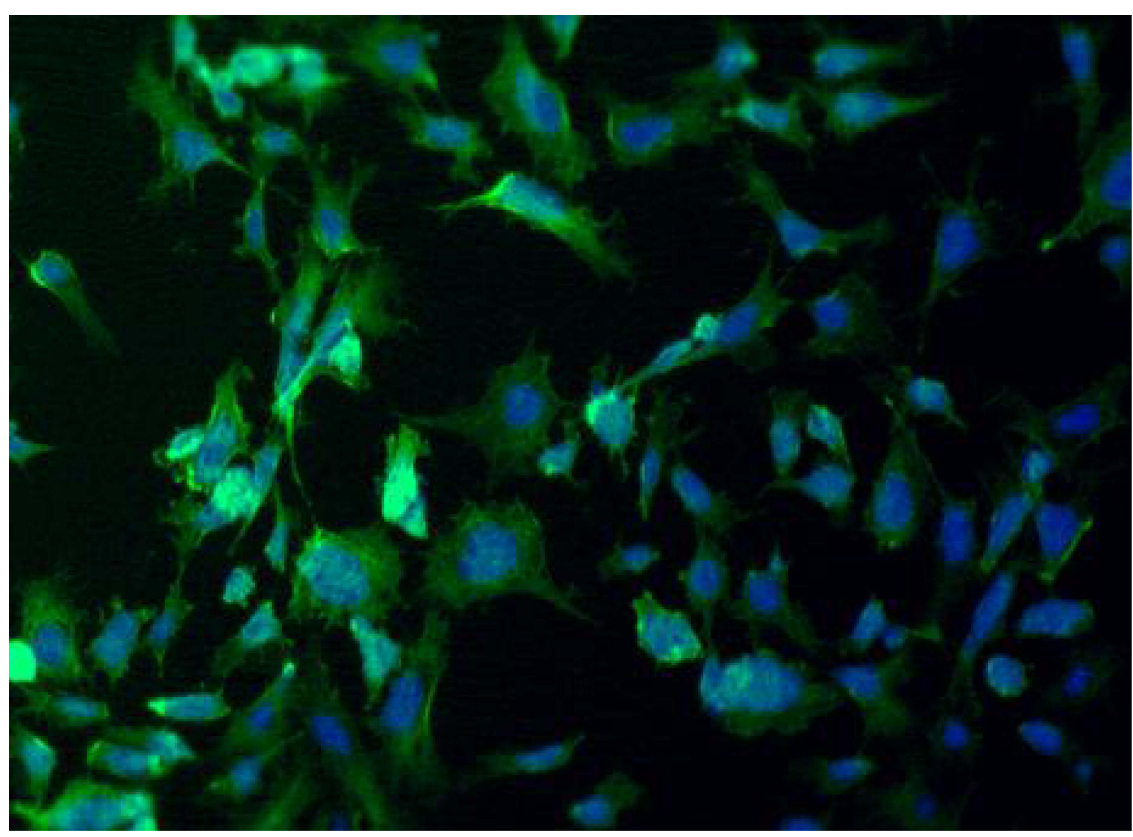

B

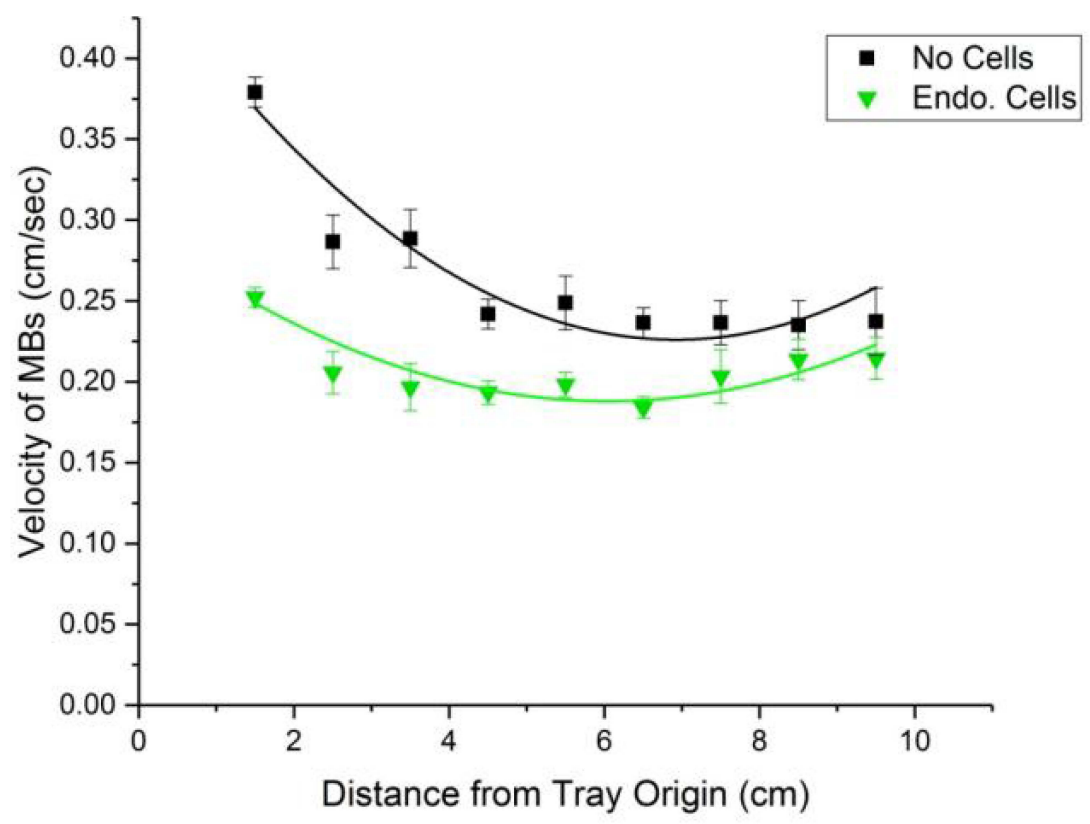

Figure 6 (A) Photomicrograph of rabbit aortic endothelial cells grown in the lanes of the MIRT tray, which were fixed then stained with Hoechst 33342 (blue) for nuclei, and phalloidin (green) for F-actin. (B) Velocity of MNPs in the MIRT tray, through PBS and over rabbit aortic endothelial cells (green), versus in PBS alone without cells (black) in the $20 \mathrm{~cm}$ offset above position $(n=6)$. 
found to be statistically significant, as shown. MNPs, therefore, were found to accelerate the fibrinolytic effect of tPA in wells, in the presence of a static magnetic field.

\section{MNP Translation and MNP/tPA Treatment in the Straight Lane MIRT Tray - Dynamic \\ Studies}

Experiments were then conducted to determine whether or not rotating MNP clusters acting alone or with the addition of tPA could cause fibrinolysis and/or whole blood clot lysis in the straight lane MIRT tray (at $37^{\circ} \mathrm{C}$ ). A photograph of a representative clot, within a lane of the straight lane MIRT tray, is shown in Figure 8A. Control lanes were set up which had clots alone in PBS, with or without the addition of IPA at the origins of the lanes. It should be noted that if tPA was added close to the clot in the MIRT tray, fibrinolysis or clot lysis would have occurred, as shown by the static experiments. Therefore, clots were made at a distance down the lane, to test whether or not MNPs could carry tPA to the clots by means of enhanced diffusion, i.e., convection. During the treatment period, the lanes were observed (and videos made) to observe for fibrinolysis and any thrombolytic effect. Clot lysis was confirmed after the experiment, by re-running MNPs down the lanes, as described in the "Methods" section. A photograph of MNPs encountering clot is seen in Figure 8B. Addition of tPA to the MNP clusters did not slow their velocities.

Neither MNPs alone, nor tPA alone (when placed at the usual starting point for the MNPs in the tray), were able to cause complete clot lysis, even after 100 min. However, when TPA and MNPs were combined, there was a lytic effect of the combination treatment on the blood clots in the MIRT tray, as seen in Figure 8C. These studies were performed in the $20 \mathrm{~cm}$ offset above position, with and without endothelial cells. With MNPs+tPA, the onset of fibrinolysis (i.e., the appearance of liquid blood) was observed at $30 \mathrm{~min}$, with $70 \mathrm{~min}$ typically being required
A

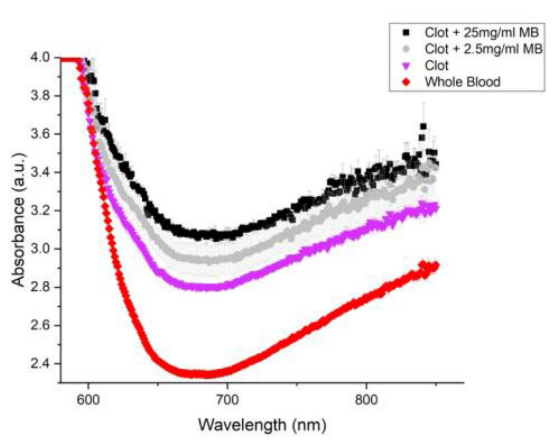

D

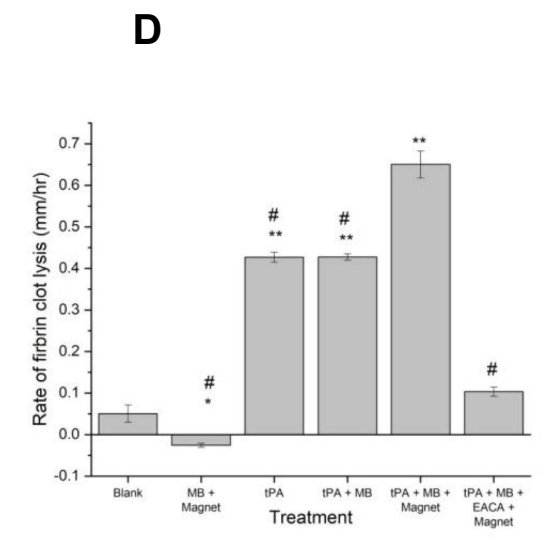

B
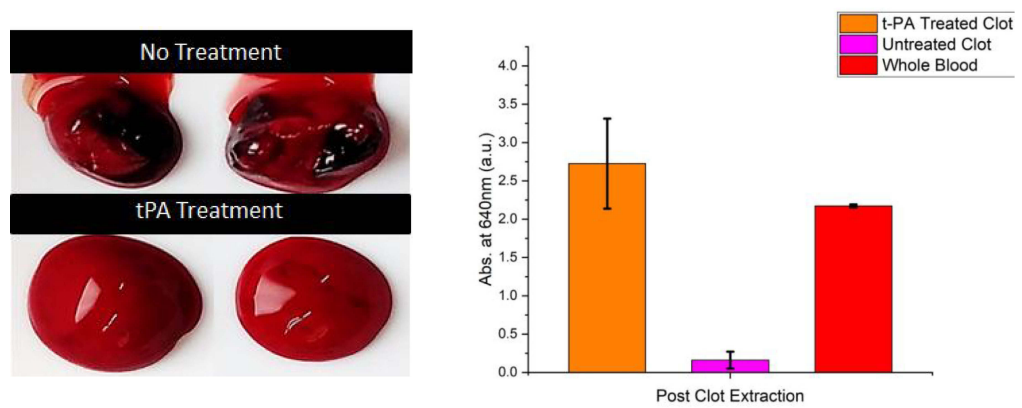

E
C

Post Clot Extraction

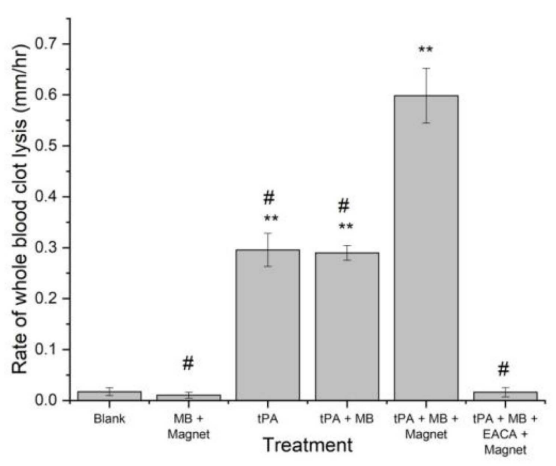

Figure 7 (A) Absorbance spectra of whole blood (red), blood clots (magenta), and blood clots with MNPs (abbreviated for brevity as "MB") at two concentrations: 2.5 mg/ $\mathrm{ml}$ (gray) and $25 \mathrm{mg} / \mathrm{ml}$ (black). (B) Photographs of untreated and tPA-treated clots after removal from the 96 -well plate. (C) Absorbance values (at $640 \mathrm{~nm}$ ) following clot extraction from the 96-well plate, $n \geq 3$. Higher values are seen with more liquid clots. (D) Rate of fibrin clot lysis (mm/hr), in response to different treatments. (E) Rate of whole blood clot lysis (mm/hr), in response to different treatments. For Panels ( $\mathbf{D}$ and $\mathbf{E}), \mathrm{n} \geq 5$. Significance is indicated as: *, $\mathrm{p}<0.05$ versus blank; **, $\mathrm{P}<0.000 \mathrm{I}$ versus blank; $\#, p<0.0001$ versus $t P A+M N P s+$ magnet. 
A

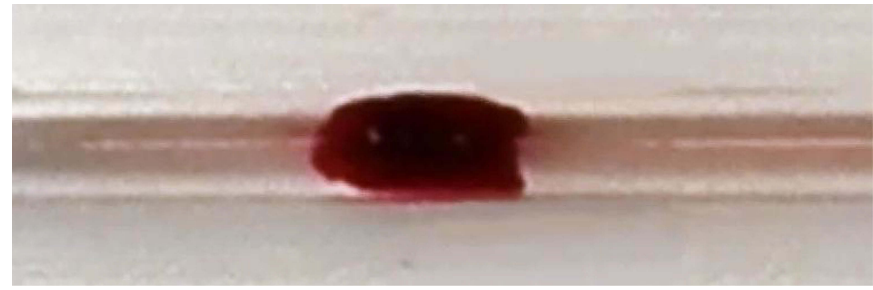

B

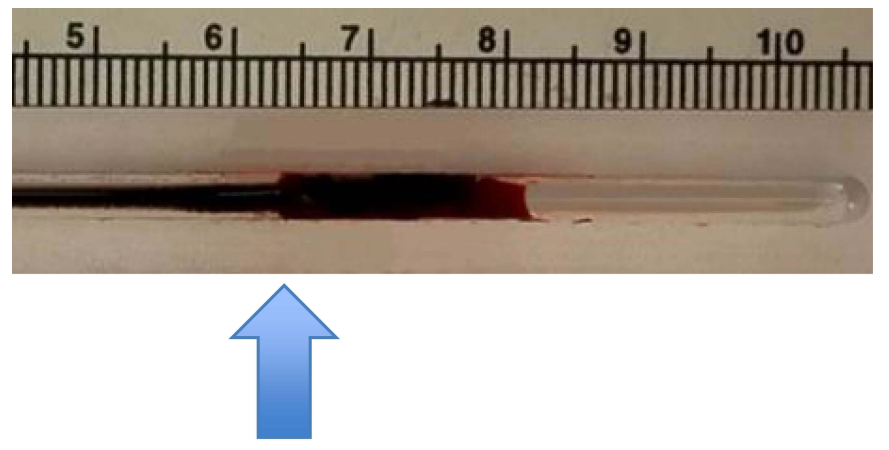

C

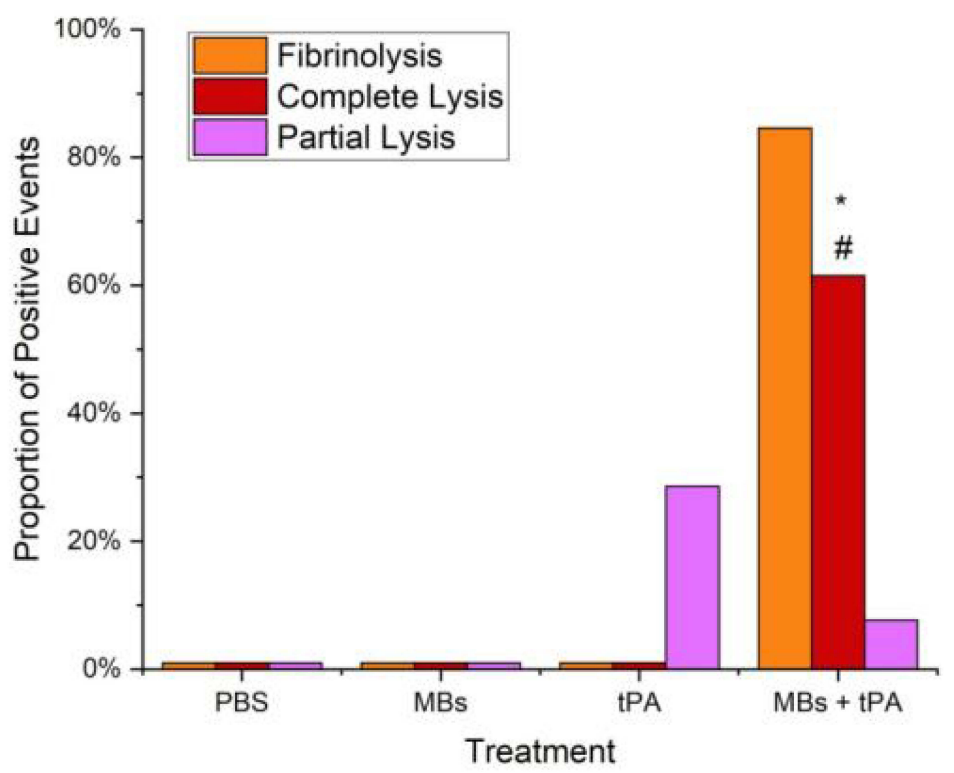

Figure 8 (A) Photograph of a blood clot produced from rabbit whole blood, and then placed within a lane of the MIRT tray. (B) MNPs (arrow) coming into contact with a blood clot, due to the translational effect of the mini-MED. (C) Occurrence of fibrinolysis and partial vs. complete clot lysis in dynamic studies within the straight lane MIRT tray, $n \geq 4$. For this assay, fibrinolysis was defined as the appearance of liquid blood and was determined independently of clot lysis. For tPA versus MNPs+tPA: *, $<<0.005$. For MNPs versus MNPs+tPA: \#, $\mathrm{p}<0.01$. 
for MNPs+tPA to create a path through the clot. Fibrinolysis was observed $85 \%$ of the time, and complete clot lysis was found in $61.5 \%$ of the MNPs+tPA experiments $(\mathrm{p}<0.01)$. Delivery of tPA to clots in the MIRT tray by MNPs also produced softer clots and morphological changes, similar to what had been seen in the "static" experiments, as described above.

\section{Discussion}

These data show that MNP clusters can be moved remotely at human-sized distances $(5-30 \mathrm{~cm})$ by a rotating permanent magnet ("mini-MED"), and channeled along straight or branched lanes of different sizes in sterilizable acrylic trays. Videography and digital analysis allowed for accurate quantification of MNP velocities. Lanes were filled with fluids of varying viscosity, including whole rabbit blood. Rabbit vascular endothelial cells were used to line the lanes of the trays, thus providing a crude model of a $1 / 8$ th inch diameter blood vessel. Thrombi were made by adding calcium chloride to whole rabbit blood, overcoming chelation by citrate. The resulting clots were transferred to 96-well plates or the MIRT tray for further study in combination with MNPs and/or tPA. The system was used to study the potential use of MNPs for delivering tPA, to enhance clot lysis. The effect of MNPs with or without tPA was first tested on clots in wells (i.e., the "static studies"). Magnetic attraction was found to enhance the fibrinolytic effect of tPA. Finally, MNPs were used to deliver tPA down the lanes of the trays to blood clots in response to the rotating magnet (i.e., the "dynamic studies"). MNPs combined with tPA were found to effectively enhance fibrinolysis at a distance. The enhanced effect of tPA occurred through drug association with MNPs, without any procedure directed at specifically binding drug to the particles themselves.

Magnetite $\left(\mathrm{Fe}_{3} \mathrm{O}_{4}\right)$, maghemite $\left(\mathrm{\gamma}-\mathrm{Fe}_{2} \mathrm{O}_{3}\right)$, and hematite $\left(\mathrm{Fe}_{2} \mathrm{O}_{3}\right)$ are all forms of iron oxide, but their properties in nanoparticle formulations are not the same. MNPs vary in magnetic properties depending on the material used. Magnetite has the strongest magnetic moment of the three, while hematite has the weakest. ${ }^{38,39}$ When ferrimagnetic particles are exposed to a magnetic field, their dipoles align and they form chain -like structures. ${ }^{40,41}$ When exposed to a rotating magnetic field, these chains further agglomerate, forming the clusters seen in our studies. Clusters of the MNPs used here counter-rotate in response to the rotating magnetic field, as they act to align their dipoles to the field. This creates a rotational force, i.e., torque. ${ }^{26,42,43}$ Interaction of the MNP clusters with a fluid, or especially a surface, creates traction and a rolling progression of the clusters. The lanes of the tray - or sides of a conduit such as a blood vessel - help to direct this rolling motion. Using serum or blood in the conduit, lining it with endothelial cells, performing tests at $37^{\circ} \mathrm{C}$, and adding branching therefore act to produce a "biomimetic model" (i.e., crude artificial blood vessel) for testing MNP movement. ${ }^{26,44,45}$

Our data indicate that the velocity at which MNP clusters move down a conduit in response to the rotating magnet (i.e., their translational movement) is dependent on factors including: 1) the characteristics of the fluid filling the conduit (notably its viscosity), 2) the nature of the walls of the conduit (especially conduit roughness; and whether or not there is a coating with cells), and 3) the position and distance of the conduit with respect to the rotating magnet. Four fluids having relevance for laboratory research and clinical applications were chosen for these studies, namely PBS, DMEM, FBS, and whole blood. Skim milk was also tested due to its intermediate viscosity value. Not unexpectedly, MNPs were found to move more slowly through fluids with higher viscosity but could be moved even through whole blood. MNPs also moved readily down the lanes of the MIRT tray lined with live endothelial cells. MNP velocities were highest when the attraction to the magnet was added to the traction along the surface caused by the rotational force. This situation was most apparent in the "pull" position close to the rotating magnet. Addition of tPA did not slow the MNP clusters.

The effect of viscosity of the transport media, while seemingly one of the simplest variables to study, is actually complex, with multiple factors to consider including Stokes' drag, rotational drag, surface friction, and dispersion of the clusters. ${ }^{40,46,47}$ Dynamic (or shear) viscosity of a fluid (designated by $\mu$ ) is a measure of the resistance of a fluid to gradually deform under shear or tensile stress. For familiar liquids, it corresponds to the perception of "thickness." Plasma viscosity is influenced by the concentrations of plasma proteins and lipoproteins, notably fibrinogen. Plasma viscosity, like lymphatic fluid, is about 1.8 $\mathrm{mPa} \cdot \mathrm{s}^{48}$ Blood viscosity is determined by plasma viscosity, hematocrit, temperature, and erythrocyte aggregation and deformability. It ranges from 3 to $5 \mathrm{mPa} \cdot \mathrm{s}$. Blood is considered a non-Newtonian fluid because it becomes less viscous at high shear rates. Cerebrospinal fluid (CSF) has a viscosity similar to water and $\mathrm{PBS}$, ranging from 0.7 to $1.0 \mathrm{mPa} \cdot \mathrm{s}$. Higher protein concentrations or cell counts do 
not significantly affect the viscosity of normal CSF. ${ }^{49,50}$ As the media becomes more viscous (due to higher protein content or interposition of blood cells, as examples), more torque is necessary to keep the MNP clusters spinning, otherwise, velocity will decrease. With increased torque, cavitation, and then heating (magnetic hyperthermia) can occur. ${ }^{51}$ Higher viscosity also imparts more friction to the clusters, which may cause them to break apart due to shear forces. ${ }^{40,41}$ The bifurcation angle, as seen in the branched tray, is yet another important hemodynamic factor. ${ }^{52-55}$ The effects of friction might be capitalized upon, however, in order to improve drug delivery at a target site. With more viscous solutions, the traction afforded by "surface walking" might not be needed at all.

Achieving an improved understanding of the factors that influence MNP agglomeration, stability, translational movement, cellular uptake, and drug binding and release will be crucial for making the jump from in vitro and animal studies, to human applications for magnetic drug targeting. ${ }^{56-60}$ Electrolyte composition, protein content, and viscosity (among many other factors) are very different in the various bodily fluids that could act as MNP conduits: cerebrospinal fluid versus blood, for instance. ${ }^{61}$ Yet to date, only a few studies have analyzed the effect of these factors on MNP movement, aggregation/agglomeration, and/or drug delivery. ${ }^{29,62}$ Critically, when nanoparticles enter a physiologic environment, they rapidly adsorb proteins and form a protein corona, which imparts a biological identity that is distinct from their synthetic identity. ${ }^{59,63,64}$ The drug delivery principle seen in our studies - that of moving unbound drug with MNP clusters may essentially be a collective corona effect. The MNP clusters are therefore functioning as medical devices (or "nanobots") for drug delivery, ${ }^{65,66}$ and do not require a mechanism for triggering drug release from the particles.

TPA is used to treat patients with vascular occlusive events such as acute ischemic stroke, and myocardial infarction. $^{7-11,67}$ Since tPA converts plasminogen to plasmin in the presence of fibrin, it has less potential to induce bleeding than other thrombolytic agents such as streptokinase and urokinase. ${ }^{14}$ Current evidence demonstrates that the sooner the tPA reaches its target in the brain's arterial system, the more favorable the outcome for the patient, i.e., "time is brain". ${ }^{20,68}$ In clinical studies, nearly half the patients did not benefit from IV tPA administration, which could certainly be due to inadequate drug delivery. ${ }^{16,69}$ Vessel recanalization rates with IV tPA for proximal arterial occlusion range from only $10-30 \%{ }^{68}$ Occlusions within the cerebral vasculature create blind alleys where drug is less likely to perfuse without magnetic targeting. Improving tPA delivery to the site of a thrombus (to promote lysis), and reducing systemic exposure (to avoid additional risk of hemorrhage), continue to be desirable goals - and would be expected to increase the efficacy and safety of tPA treatment for stroke and other thrombotic disorders.

Meta-analyses of clinical trials investigating the efficacy of endovascular therapies for stroke (and retrospective analyses of patient databases) have provided strong evidence to support the use of catheter-based thrombectomy within $6 \mathrm{hrs}$ of stroke onset, in selected patient subpopulations, irrespective of receipt of intravenous tPA. $^{20}$ Despite inherent risks, endovascular therapy promises higher recanalization rates than IV tPA, and potentially longer treatment windows. ${ }^{68}$ We would expect that if MNPs were used to enhance tPA delivery clinically, they would be delivered by an endovascular route. Mechanical thrombectomy already requires threading of a catheter up to the vicinity of the blocked artery, but is not always successful, and cannot always access even more distal small vessel occlusions. The safety of administering tPA with MNPs locally needs to be proven but would be expected to incur fewer systemic side-effects, such as the hypotension observed to occur with IV coadministration. ${ }^{33}$ Combination (or sequential) therapy, for instance, utilizing tPA and pro-urokinase, ${ }^{70,71}$ may make MNP therapy for clot lysis even more effective.

The application of nanotechnology to vascular disease is still an emerging field, but one which holds great promise. $^{16,23,72}$ There have been several reports of tPA and other thrombolytic drugs being bound to various types of nanoparticles, including iron oxide nanoparticles, with characterization and testing in vitro and in rodents using non-rotating magnets..$^{22,57,73-84}$ It has recently been reported that polyacrylic acid - coated tPA - MNPs have been successfully tested in vitro and in a mouse model of embolic stroke, in which a rotating magnetic field was employed. ${ }^{21}$ TPA has also been transported to blood clots in vitro and to clots in mice femoral arteries, using a rotating magnetic system. ${ }^{66}$ Our findings are consistent with these results, but consider different dimensions, and factors (such as media and endothelial cells) which affect the rotation and translation of the MNP clusters. As of the submission of this manuscript, a PubMed search of "nanoparticles and thrombolysis" revealed no results for clinical trials, underscoring the need for developing improved 
models so that the gap between laboratory and successful clinical use can finally be bridged.

\section{Conclusions}

Despite advances with IV TPA in the endovascular treatment of stroke and other thrombotic disorders, these diseases continue to cause an unacceptable degree of disability and death worldwide. Further progress in delivering drug to the site of the thrombus needs to be made. Animal studies, while important, do not test human-sized distances, which is critically important for predicting the success of magnetic drug targeting. Rotating magnetic systems offer an improved way to move magnetic particles at clinically relevant distances, since the MNPs can walk along physiologic surfaces, such as the endothelial linings of blood vessels. ${ }^{29,65}$

In studies reported here, MNP clusters functioned as devices ("micro-motors") for improving drug delivery, eliminating the need for adrug release mechanism. While these model components are rough prototypes, the principles they establish set the stage for more sophisticated studies of MNP behavior in vitro, before proceeding to animal studies and clinical trials. Next-generation tray design could include flow, pulsations, and more realistic branching patterns based on data from individual patients. Our results indicate that MNPs should be studied further for use in augmenting the delivery of tPA in the treatment of stroke and other vascular occlusive diseases.

\section{Acknowledgments}

The authors would like to thank Zack Gaertner and Adam Levin for being early proponents of this project, Dylan Lynch and Joel Rodriguez of the University of Illinois at Chicago for their assistance in milling the MIRT trays, Michelle Van Scoyk of the University of Illinois Cancer Center for assistance with microscopy, Brian Kidd and Kay Broschat from Pulse Therapeutics, Inc., and Dr. Michael Tones (also from Pulse) for a critical review of the manuscript. Data reported here were presented at the Annual Meeting of the Society for Neuroscience, Washington, D.C., November 2017.

\section{Disclosure}

Mr Sebastian P Pernal and Mr Alexander J Willis report gift support from Pulse Therapeutics, Inc. to the University of Illinois at Chicago during the conduct of the study. $\mathrm{Mr}$ Michael E Sabo reports grants from the National Institutes of Health, during the conduct of the study; he was an employee of Pulse Therapeutics Inc., during the submitted work. Ms Laura M Moore was an employee of Pulse Therapeutics, Inc. Mr Sean C Morris reports personal fees from Pulse Therapeutics, Inc., he also is an employee of Pulse Therapeutics Inc.. In addition, he has a patent 8715150 issued, a patent 9339664 issued, a patent 10029008 issued, a patent 8308628 issued, a patent 8313422 issued, a patent 8529428 issued, a patent 8926491 issued, a patent 9345498 issued, a patent 10159734 issued, a patent 9883878 issued, a patent 2010313105 issued, a patent 2777841 issued, a patent 2934401 issued, a patent ZL 201080060370.4 issued, a patent EP 2496302 issued, a patent 219515 issued, a patent 602010058639.1 issued, a patent 246714 issued, a patent 5865252 issued, a patent 6169677 issued, a patent 10-1616465 issued, a patent 324087 issued, a patent 2012/02947 issued, a patent EP 2849657 issued, a patent 602013042824.7 issued, a patent 6335163 issued, a patent 2019/0038748 pending, a patent 2019/0255174 pending, a patent 2018/0221041 pending, a patent WO/2011/053984 issued, a patent WO/2013/ 173235 issued, and a patent 15/886, 130 issued. Dr Francis $M$ Creighton reports grants from NIH/NINDS, and grants from NIH/NIMHD, during the conduct of the study. In addition, he has a patent US10159734 issued, a patent US10029008 issued, a patent US9883878 issued, a patent US9345498 issued, a patent US8926491 issued, a patent US8715150 issued, a patent US8529428 issued, a patent US8313422 issued, and a patent US8308628 issued. Dr Herbert H Engelhard reports gifts and non-financial support, from Pulse Therapeutics, Inc. made to the University of Illinois at Chicago, during the conduct of the study. In addition, he has a patent "Tissue Culture Tray and System for the In Vitro Testing and Characterization of MagneticallyInduced Rotation and Translational Motion of Magnetic Particles" July 2017 US20180024109A1 pending. The authors report no other conflicts of interest in this work.

\section{References}

1. ISTH Steering. Committee for World Thrombosis Day. Thrombosis: a major contributor to the global disease burden. J Thromb Haemost. 2014;12:1580-1590. doi:10.1111/jth.12698

2. Kumar SS, Sabu A. Fibrinolytic enzymes for thrombolytic therapy. $A d v$ Exp Med Biol. 2019;1148:345-381. doi:10.1007/978-981-13-7709-9_15

3. Borohovitz A, Weinberg MD, Weinberg I. Pulmonary embolism: care standards in 2018. Prog Cardiovasc Dis. 2018;60:613-621. doi:10.1016/j.pcad.2017.12.005

4. Hess CN, Hiatt WR. Antithrombotic therapy for peripheral artery disease in 2018. JAMA. 2018;319:2329-2330. doi:10.1001/jama. 2018.5422

5. Engelhard HH, Cozzens JW, Green D. Deep venous thrombosis and pulmonary embolism. Perspect Neurol Surg. 1997;8(1):1-17. 
6. Benjamin EJ, Blaha MJ, Chiuve SE, et al. Heart disease and stroke statistics-2017 update: a report from the American Heart Association. Circulation. 2017;135:e146-603. doi:10.1161/CIR.00000000000004 85

7. Xiong Y, Manwani B, Fisher M. Management of acute ischemic stroke. Am J Med. 2019;132:286-291. doi:10.1016/j.amjmed.2018. 10.019

8. Chapman SN, Mehndiratta P, Johansen MC, McMurry TL, Johnston KC, Southerland AM. Current perspectives on the use of intravenous recombinant tissue plasminogen activator (tPA) for treatment of acute ischemic stroke. Vasc Health Risk Manag. 2014;10:75-87. doi:10.2147/VHRM.S39213

9. Hatcher MA, Starr JA. Role of tissue plasminogen activator in acute ischemic stroke. Ann Pharmacother. 2011;45:364-371. doi:10.1345/ aph.1P525

10. Marshall RS. Progress in intravenous thrombolytic therapy for acute stroke. JAMA Neurol. 2015;72:928-934. doi:10.1001/jamaneurol. 2015.0835

11. Brorson JR, Cifu AS. Management of patients with acute ischemic stroke. JAMA. 2019;322:777. doi:10.1001/jama.2019.10436

12. Jilani TN, Siddiqui AH. Tissue Plasminogen Activator. StatPearls, Treasure Island (FL): StatPearls Publishing; 2019.

13. El-Sherbiny IM, Elkholi IE, Yacoub MH. Tissue plasminogen activator-based clot busting: controlled delivery approaches. Glob Cardiol Sci Pract. 2014;2014:336-349. doi:10.5339/gcsp.2014.46

14. Rogers SD, Riemersma LB, Clements SD. Tissue plasminogen activator: an evaluation of clinical efficacy in acute myocardial infarction. Pharmacotherapy. 1987;7:111-121. doi:10.1002/j.18759114.1987.tb04035.x

15. Lippi G, Mattiuzzi C, Favaloro EJ. Novel and emerging therapies: thrombus-targeted fibrinolysis. Semin Thromb Hemost. 2013;39:48-58. doi:10.1055/s-0032-1328935

16. Liu S, Feng X, Jin R, Li G. Tissue plasminogen activator-based nanothrombolysis for ischemic stroke. Expert Opin Drug Deliv. 2018;15:173-184. doi:10.1080/17425247.2018.1384464

17. Panagiotou S, Saha S. Therapeutic benefits of nanoparticles in stroke. Front Neurosci. 2015;9:182. doi:10.3389/fnins.2015.00182

18. Clements M, Bonnecaze R. Diffusion limited delivery of streptokinase and tPA to a thrombus. Cardiovasc Eng Technol. 2013;4:364-373. doi:10.1007/s13239-013-0160-8

19. Riedel CH, Zimmermann P, Jensen-Kondering U, Stingele R, Deuschl G, Jansen O. The importance of size: successful recanalization by intravenous thrombolysis in acute anterior stroke depends on thrombus length. Stroke. 2011;42:1775-1777. doi:10.1161/ STROKEAHA.110.609693

20. Saver JL, Goyal M, van der Lugt A, et al. Time to treatment with endovascular thrombectomy and outcomes from ischemic stroke: a meta-analysis. JAMA. 2016;316:1279-1288. doi:10.1001/jama.2016. 13647

21. Huang L, Wang J, Huang S, Siaw-Debrah F, Nyanzu M, Zhuge Q. Polyacrylic acid-coated nanoparticles loaded with recombinant tissue plasminogen activator for the treatment of mice with ischemic stroke. Biochem Biophys Res Commun. 2019;516:565-570. doi:10.1016/j. bbrc.2019.06.079

22. Tadayon A, Jamshidi R, Esmaeili A. Delivery of tissue plasminogen activator and streptokinase magnetic nanoparticles to target vascular diseases. Int J Pharm. 2015;495:428-438. doi:10.1016/j.ijpharm.2015.09.008

23. Gupta MK, Lee Y, Boire TC, Lee J-B, Kim WS, Sung H-J. Recent strategies to design vascular theranostic nanoparticles. Nanotheranostics. 2017;1:166-177. doi:10.7150/ntno.18531

24. Shcharbina N, Shcharbin D, Bryszewska M. Nanomaterials in stroke treatment: perspectives. Stroke. 2013;44:2351-2355. doi:10.1161/ STROKEAHA.113.001298

25. Bonnecaze R, Clements M. Magnetically driven flows of suspensions of rods to deliver clot-busting drugs to dead-end arteries. Bull Am Phys Soc. 2014;59:D9.7.
26. Karle M, Wöhrle J, Miwa J, et al. Controlled counter-flow motion of magnetic bead chains rolling along microchannels. Microfluid Nanofluidics. 2011;10:935-939. doi:10.1007/s10404-010-0727-8

27. Engelhard HH, Petruska DA. Imaging and movement of iron-oxidebound antibody microparticles in brain and cerebrospinal fluid. Cancer Biochem Biophys. 1992;13:1-12.

28. Pouponneau P, Leroux J-C, Martel S. Magnetic nanoparticles encapsulated into biodegradable microparticles steered with an upgraded magnetic resonance imaging system for tumor chemoembolization. Biomaterials. 2009;30:6327-6332. doi:10.1016/j.biomaterials.2009.08.005

29. Engelhard HH, Pernal SP, Gaertner ZA, et al. A novel tissue culture tray for the study of magnetically induced rotation and translation of iron oxide nanoparticles. IEEE Magnet Lett. 2017;8:1-5. doi:10.1109/LMAG.2017.2761818

30. Liu JF, Jang B, Issadore D, Tsourkas A. Use of magnetic fields and nanoparticles to trigger drug release and improve tumor targeting. Wiley Interdiscip Rev Nanomed Nanobiotechnol. 2019;11:e1571. doi:10.1002/wnan.1571

31. Engelhard H, Gaertner Z, Levin A, et al. Rotating Magnetic Beads for Enhanced Drug Delivery: Characterization of Bead Velocity, Imaging, and Adherence to Cellular Monolayers, Abstract 3104. American Association for Cancer Research; 2017. doi:10.1158/ 1538-7445.AM2017-3104

32. Pernal SP, Willis AJ, Engelhard HH. Magnetic Nanoparticles (MNPS) for Cancer Drug Delivery: The Value of in vitro Modeling, Abstract 4661.American Association for Cancer Research; 2018:4661. doi:10.1158/1538-7445.AM2018-4661

33. Derdeyn C, Campbell B, Bladin C, et al. Magnetic particle surface characteristics associated with bradykinin upregulation: cause of hypotension in the magnetically-enhanced diffusion (MED) of intravenous tPA in acute ischemic stroke feasibility trial, Abstract W P404. Stroke. 2015;46 Suppl 1:AWP404.

34. Bladin C, Levi C, Parsons M, et al. Can we augment stroke thrombolysis? The use of magnetically-enhanced diffusion $\left(\mathrm{MED}^{\mathrm{TM}}\right)$ of intravenous tPA in acute ischemic stroke. Int $J$ Stroke. 2015;10 (3):18-19.

35. Prasad S, Kashyap RS, Deopujari JY, Purohit HJ, Taori GM, Daginawala HF. Development of an in vitro model to study clot lysis activity of thrombolytic drugs. Thromb J. 2006;4:14. doi:10.1186/1477-9560-4-14

36. Viennet C, Laurensou C, Goydadin AC, Faivre B, Muret P, Humbert P. Development of an in vitro fibrin clot model to evaluate fibrinolytic agents for wound care application. $J$ Wound Care. 2014;23:66-7, 70, 72. doi:10.12968/jowc.2014.23.2.66

37. Mann KG, Whelihan MF, Butenas S, Orfeo T. Citrate anticoagulation and the dynamics of thrombin generation. J Thromb Haemost. 2007;5:2055-2061. doi:10.1111/j.1538-7836.2007.02710.x

38. Lee GP, Pernal SP, Shokuhfar T, Engelhard HH. Nanoparticles as therapeutic agents for patients with brain tumors. In: Newton $\mathrm{H}$, editor. Handbook of Brain Tumor Chemotherapy, Molecular Therapeutics and Immunotherapy. 2nd ed. San Diego: Academic Press; 2018:229-246.

39. Teja AS, Koh P-Y. Synthesis, properties, and applications of magnetic iron oxide nanoparticles. Prog Crys Growth Charact Mater. 2009;55:22-45. doi:10.1016/j.pcrysgrow.2008.08.003

40. Ganguly R, Puri IK. Microfluidic transport in magnetic MEMS and bioMEMS. Wiley Interdiscip Rev Nanomed Nanobiotechnol. 2010;2:382-399. doi:10.1002/wnan.92

41. Vuppu AK, Garcia AA, Hayes MA. Video microscopy of dynamically aggregated paramagnetic particle chains in an applied rotating magnetic field. Langmuir. 2003;19:8646-8653. doi:10.1021/ la034195a

42. Cantillon-Murphy P, Wald LL, Adalsteinsson E, Zahn M. Simulating magnetic nanoparticle behavior in low-field MRI under transverse rotating fields and imposed fluid flow. J Magn Magn Mater. 2010;322:2607-2617. doi:10.1016/j.jmmm.2010.03.029 
43. Rikken RSM, Nolte RJM, Maan JC, van Hest JCM, Wilson DA, Christianen PCM. Manipulation of micro- and nanostructure motion with magnetic fields. Soft Matter. 2014;10:1295-1308. doi:10.1039/ c3sm52294f

44. Shinohara S, Kihara T, Sakai S, et al. Fabrication of in vitro three-dimensional multilayered blood vessel model using human endothelial and smooth muscle cells and high-strength PEG hydrogel. J Biosci Bioeng. 2013;116:231-234. doi:10.1016/j.jbiosc.2013.02.013

45. Thomas A, Daniel Ou-Yang H, Lowe-Krentz L, Muzykantov VR, Liu Y. Biomimetic channel modeling local vascular dynamics of pro-inflammatory endothelial changes. Biomicrofluidics. 2016;10:014101. doi:10.1063/1.4936672

46. Nacev A, Beni C, Bruno O, Shapiro B. Magnetic nanoparticle transport within flowing blood and into surrounding tissue. Nanomedicine (Lond). 2010;5:1459-1466. doi:10.2217/nnm.10.104

47. Nacev A, Beni C, Bruno O, Shapiro B. The behaviors of ferro-magnetic nano-particles in and around blood vessels under applied magnetic fields. J Magn Magn Mater. 2011;323:651-668. doi:10.1016/j. jmmm.2010.09.008

48. Bouta EM, Wood RW, Brown EB, Rahimi H, Ritchlin CT, Schwarz EM. In vivo quantification of lymph viscosity and pressure in lymphatic vessels and draining lymph nodes of arthritic joints in mice. J Physiol (Lond). 2014;592:1213-1223. doi:10.1113/jphysiol. 2013.266700

49. Brydon HL, Hayward R, Harkness W, Bayston R. Physical properties of cerebrospinal fluid of relevance to shunt function. 1: the effect of protein upon CSF viscosity. Br J Neurosurg. 1995;9:639-644. doi: $10.1080 / 02688699550040927$

50. Bloomfield IG, Johnston IH, Bilston LE. Effects of proteins, blood cells and glucose on the viscosity of cerebrospinal fluid. Pediatr Neurosurg. 1998;28:246-251. doi:10.1159/000028659

51. Golovin YI, Gribanovsky SL, Golovin DY, et al. Towards nanomedicines of the future: remote magneto-mechanical actuation of nanomedicines by alternating magnetic fields. $J$ Control Release. 2015;219:43-60. doi:10.1016/j.jconrel.2015.09.038

52. Lou Z, Yang WJ. Biofluid dynamics at arterial bifurcations. Crit Rev Biomed Eng. 1992;19:455-493.

53. Yang J, Pak YE, Lee T-R. Predicting bifurcation angle effect on blood flow in the microvasculature. Microvasc Res. 2016;108:22-28. doi:10.1016/j. mvr.2016.07.001

54. Chiastra C, Iannaccone F, Grundeken MJ, et al. Coronary fractional flow reserve measurements of a stenosed side branch: a computational study investigating the influence of the bifurcation angle. Biomed Eng Online. 2016;15:91. doi:10.1186/s12938-016-0211-0

55. Rashad S, Sugiyama S-I, Niizuma K, et al. Impact of bifurcation angle and inflow coefficient on the rupture risk of bifurcation type basilar artery tip aneurysms. J Neurosurg. 2018;128:723-730. doi:10.3171/2016.10.JNS161695

56. García-Jimeno S, Estelrich J, Callejas-Fernández J, Roldán-Vargas S. Reversible and irreversible aggregation of magnetic liposomes. Nanoscale. 2017;9:15131-15143. doi:10.1039/c7nr05301k

57. Friedrich RP, Zaloga J, Schreiber E, et al. Tissue plasminogen activator binding to superparamagnetic iron oxide nanoparticle-covalent versus adsorptive approach. Nanoscale Res Lett. 2016;11:297. doi:10.1186/s11671-016-1521-7

58. Walkey CD, Olsen JB, Guo H, Emili A, Chan WCW. Nanoparticle size and surface chemistry determine serum protein adsorption and macrophage uptake. $J$ Am Chem Soc. 2012;134:2139-2147. doi:10.1021/ja2084338

59. Walkey CD, Chan WCW. Understanding and controlling the interaction of nanomaterials with proteins in a physiological environment Chem Soc Rev. 2012;41:2780-2799. doi:10.1039/c1cs15233e

60. Mazuel F, Espinosa A, Luciani N, et al. Massive intracellular biodegradation of iron oxide nanoparticles evidenced magnetically at single-endosome and tissue levels. ACS Nano. 2016;10:7627-7638. doi:10.1021/acsnano.6b02876
61. Windberger U, Bartholovitsch A, Plasenzotti R, Korak KJ, Heinze G. Whole blood viscosity, plasma viscosity and erythrocyte aggregation in nine mammalian species: reference values and comparison of data. Exp Physiol. 2003;88:431-440. doi:10.1113/eph8802496

62. Etheridge ML, Hurley KR, Zhang J, et al. Accounting for biological aggregation in heating and imaging of magnetic nanoparticles. Technology (Singap World Sci). 2014;2:214-228. doi:10.1142/ S2339547814500198

63. Lundqvist M, Stigler J, Elia G, Lynch I, Cedervall T, Dawson KA Nanoparticle size and surface properties determine the protein corona with possible implications for biological impacts. Proc Natl Acad Sci USA. 2008;105:14265-14270. doi:10.1073/pnas.0805135105

64. Tenzer S, Docter D, Rosfa S, et al. Nanoparticle size is a critical physicochemical determinant of the human blood plasma corona: a comprehensive quantitative proteomic analysis. ACS Nano. 2011;5:7155-7167. doi:10.1021/nn201950e

65. Fountain TWR, Kailat PV, Abbott JJ. Wireless Control of Magnetic Helical Microrobots Using a Rotating-Permanent-Magnet Manipulator. IEEE; 2010: 576-581. doi:10.1109/ROBOT.2010.5509245

66. Cheng R, Huang W, Huang L, et al. Acceleration of tissue plasminogen activator-mediated thrombolysis by magnetically powered nanomotors. ACS Nano. 2014;8:7746-7754. doi:10.1021/nn5029955

67. O'Gara PT, Kushner FG, Ascheim DD, et al. ACCF/AHA guideline for the management of ST-elevation myocardial infarction: executive summary: a report of the American College of Cardiology Foundation/American Heart Association Task Force on Practice Guidelines: developed in collaboration with the American College of Emergency Physicians and Society for Cardiovascular Angiography and Interventions. Catheter Cardiovasc Interv. 2013;2013(82):E1-E27. doi:10.1002/ccd.24776

68. Killer M, Ladurner G, Kunz AB, Kraus J. Current endovascular treatment of acute stroke and future aspects. Drug Discov Today. 2010;15:640-647. doi:10.1016/j.drudis.2010.04.007

69. Menon BK, Al-Ajlan FS, Najm M, et al. Association of clinical, imaging, and thrombus characteristics with recanalization of visible intracranial occlusion in patients with acute ischemic stroke. JAMA. 2018;320:1017-1026. doi:10.1001/jama.2018.12498

70. Gurewich V. Thrombolysis: a critical first-line therapy with an unfulfilled potential. Am J Med. 2016;129:573-575. doi:10.1016/j. amjmed.2015.11.033

71. Pannell R, Li S, Gurewich V. Fibrin-specific and effective clot lysis requires both plasminogen activators and for them to be in a sequential rather than simultaneous combination. J Thromb Thrombolysis. 2017;44:210-215. doi:10.1007/s11239-017-1514-0

72. Wickline SA, Neubauer AM, Winter P, Caruthers S, Lanza G. Applications of nanotechnology to atherosclerosis, thrombosis, and vascular biology. Arterioscler Thromb Vasc Biol. 2006;26:435-441. doi:10.1161/01.ATV.0000201069.47550.8b

73. Yang H-W, Hua M-Y, Lin K-J, et al. Bioconjugation of recombinant tissue plasminogen activator to magnetic nanocarriers for targeted thrombolysis. Int J Nanomedicine. 2012;7:5159-5173. doi:10.2147/ IJN.S32939

74. Ma Y-H, Wu S-Y, Wu T, Chang Y-J, Hua M-Y, Chen J-P. Magnetically targeted thrombolysis with recombinant tissue plasminogen activator bound to polyacrylic acid-coated nanoparticles. Biomaterials. 2009;30:3343-3351. doi:10.1016/j.biomaterials.2009.02.034

75. Wang M, Zhang J, Yuan Z, Yang W, Wu Q, Gu H. Targeted thrombolysis by using of magnetic mesoporous silica nanoparticles. J Biomed Nanotechnol. 2012;8:624-632. doi:10.1166/jbn.2012.1416

76. Zhong Y, Gong WJ, Gao XH, et al. Synthesis and evaluation of a novel nanoparticle carrying urokinase used in targeted thrombolysis. J Biomed Mater Res A. 2019. doi:10.1002/jbm.a.36803

77. Zhou J, Guo D, Zhang Y, Wu W, Ran H, Wang Z. Construction and evaluation of $\mathrm{Fe}_{3} \mathrm{O}_{4}$-based PLGA nanoparticles carrying rtPA used in the detection of thrombosis and in targeted thrombolysis. ACS Appl Mater Interfaces. 2014;6:5566-5576. doi:10.1021/am406008k 
78. Prilepskii AY, Fakhardo AF, Drozdov AS, et al. Urokinaseconjugated magnetite nanoparticles as a promising drug delivery system for targeted thrombolysis: synthesis and preclinical evaluation. ACS Appl Mater Interfaces. 2018;10:36764-36775. doi:10.1021/acsami.8b14790

79. Heid S, Unterweger H, Tietze R, et al. Synthesis and characterization of tissue plasminogen activator-functionalized superparamagnetic iron oxide nanoparticles for targeted fibrin clot dissolution. Int J Mol Sci. 2017:18. doi:10.3390/ijms18091837.

80. Chang M, Lin Y-H, Gabayno JL, Li Q, Liu X. Thrombolysis based on magnetically-controlled surface-functionalized Fe3O4 nanoparticle. Bioengineered. 2017;8:29-35. doi:10.1080/21655979.2016.1227145
81. Ma Y-H, Liu C-H, Liang Y, Chen J-P WT. Targeted delivery of plasminogen activators for thrombolytic therapy: an integrative evaluation. Molecules. 2019;24. doi:10.3390/molecules24183407

82. Shamsi M, Zahedi P. On-chip preparation of streptokinase entrapped in chitosan nanoparticles used in thrombolytic therapy potentially. J Pharm Sci. 2017;106:3623-3630. doi:10.1016/j.xphs.2017.08.001

83. Chen JP, Yang PC, Ma YH, Tu SJ, Lu YJ. Targeted delivery of tissue plasminogen activator by binding to silica-coated magnetic nanoparticle. Int J Nanomedicine. 2012;7:5137-5149. doi:10.2147/IJN.S36197

84. Disharoon D, Marr DWM, Neeves KB. Engineered microparticles and nanoparticles for fibrinolysis. $J$ Thromb Haemost. 2019;17:2004-2015. doi:10.1111/jth.14637

\section{Publish your work in this journal}

The International Journal of Nanomedicine is an international, peerreviewed journal focusing on the application of nanotechnology in diagnostics, therapeutics, and drug delivery systems throughout the biomedical field. This journal is indexed on PubMed Central, MedLine, CAS, SciSearch ${ }^{\mathbb{R}}$, Current Contents ${ }^{\mathbb{R}} /$ Clinical Medicine, $^{-}$
Journal Citation Reports/Science Edition, EMBase, Scopus and the Elsevier Bibliographic databases. The manuscript management system is completely online and includes a very quick and fair peer-review system, which is all easy to use. Visit http://www.dovepress.com/ testimonials.php to read real quotes from published authors. 